\title{
Emergence of active topological glass through directed chain dynamics and nonequilibrium phase segregation
}

\author{
Iurii Chubak $\odot,{ }^{1, *}$ Christos N. Likos $\odot,{ }^{1, \dagger}$ Kurt Kremer $\odot,{ }^{2, \ddagger}$ and Jan Smrek $\oplus^{1, \S}$ \\ ${ }^{1}$ Faculty of Physics, University of Vienna, Boltzmanngasse 5, A-1090 Vienna, Austria \\ ${ }^{2}$ Max Planck Institute for Polymer Research, Ackermannweg 10, 55128 Mainz, Germany
}

(Received 10 August 2020; accepted 22 October 2020; published 18 November 2020)

\begin{abstract}
Active matter states defy many notions that have been established for systems in thermodynamic equilibrium. Nevertheless, the lack of detailed balance might be utilized to design nonequilibrium materials with unique properties. Recently we have shown, employing a model of ring polymers containing segments with a larger mobility than given by equilibrium thermal fluctuations, that making polymers with intrinsic topology active can result in states that relax extremely slowly, the so-called active topological glass. In this paper, we focus on the role of nonequilibrium phase separation in the vitrification process. In particular, we detail the polymer dynamics and show that such activity-driven glassy states arise from heterogeneity of segmental dynamics that emerges on all scales. Provided that the activity quench is strong enough, the rings feature an oriented reptationlike motion, with the active segment serving as an effective chain's end, resulting into a dramatic increase of inter-ring treading that vitrifies the system. The scaling properties of the ensuing steady-state ring conformations, which are significantly elongated and usually possess a doubly folded structure, are discussed and compared to equilibrium counterparts. We further examine the connection between the glass formation and the nonequilibrium phase separation and we find that both appear to be initiated by the contrasting dynamics of ring segments. Finally, we consider the effect of nonequilibrium phase separation in other active copolymer architectures.
\end{abstract}

DOI: 10.1103/PhysRevResearch.2.043249

\section{INTRODUCTION}

Properties of ring polymer systems, albeit having been the subject of longstanding theoretical and experimental research, have not yet been understood in their entirety. The topology of each ring is fixed at the synthesis and the noncrossability of the chains gives rise to unique scaling and material properties that range from individual chains [1] to solutions and melts [2-8]. Furthermore, in contrast to other polymer architectures, the mathematical difficulties to capture topological constraints, even in the simplest problem of two interacting unknotted loops [9], make it a suitable problem for exploration and effective description with the aid of computer simulations [10-14]. The semidilute and melt conditions that are relevant in biological applications $[15,16]$ present an even greater modeling challenge because many topological constraints of overlapping chains are not pairwise additive [17] and have to be satisfied simultaneously. Due to involved mathematical complexity despite the simple intuitive rule of fixed topology, extensive studies focused on the prototypical

\footnotetext{
*iurii.chubak@univie.ac.at

†christos.likos@univie.ac.at

*kremer@mpip-mainz.mpg.de

$\S$ jan.smrek@univie.ac.at
}

Published by the American Physical Society under the terms of the Creative Commons Attribution 4.0 International license. Further distribution of this work must maintain attribution to the author(s) and the published article's title, journal citation, and DOI. example of the melt of unknotted and nonconcatenated rings. Yet, many fundamental questions remain open.

One such question pertaining from the beginnings of the field is the existence of topological glass [18]. The rings cannot cross but can thread each other by piercing through one another's openings. The threading restricts relative ring motion and prolongs their relaxation [19-21]. In melt conditions where many rings overlap, a hierarchical network of threadings would generate a glassy state when relaxation of a ring is conditioned on the sequence of relaxation of other rings. This conjectured type of glass arises without the change in volume fraction or temperature, the control parameter being the ring length, which impacts the propensity of mutual ring threading. The concept of topological glass has been recently put to test by so-called pinning perturbations of the melt [22,23]. When a fraction of all rings is artificially immobilized, the whole system exhibits glassy characteristics. The longer the rings, the smaller the fraction of pinned rings sufficient to observe the topological glass. The extrapolated ring length for the zero pinned rings fraction is beyond the current reach of both experiment and simulation. In these works, the unpinned rings are in equilibrium and, as such, exhibit threading of their neighbors that is limited by (i) the entropically accessible conformations and (ii) the number of the neighbors, which saturates in the long ring limit due to the compact conformation of the rings [3]. Therefore, other ideas to enhance the threading of shorter rings and create a topological glass have been looked for.

Recently, we have shown [24] that a state of topological glass can be attained by rings of moderate length, provided that their segments are subject to heterogeneous activity. The 

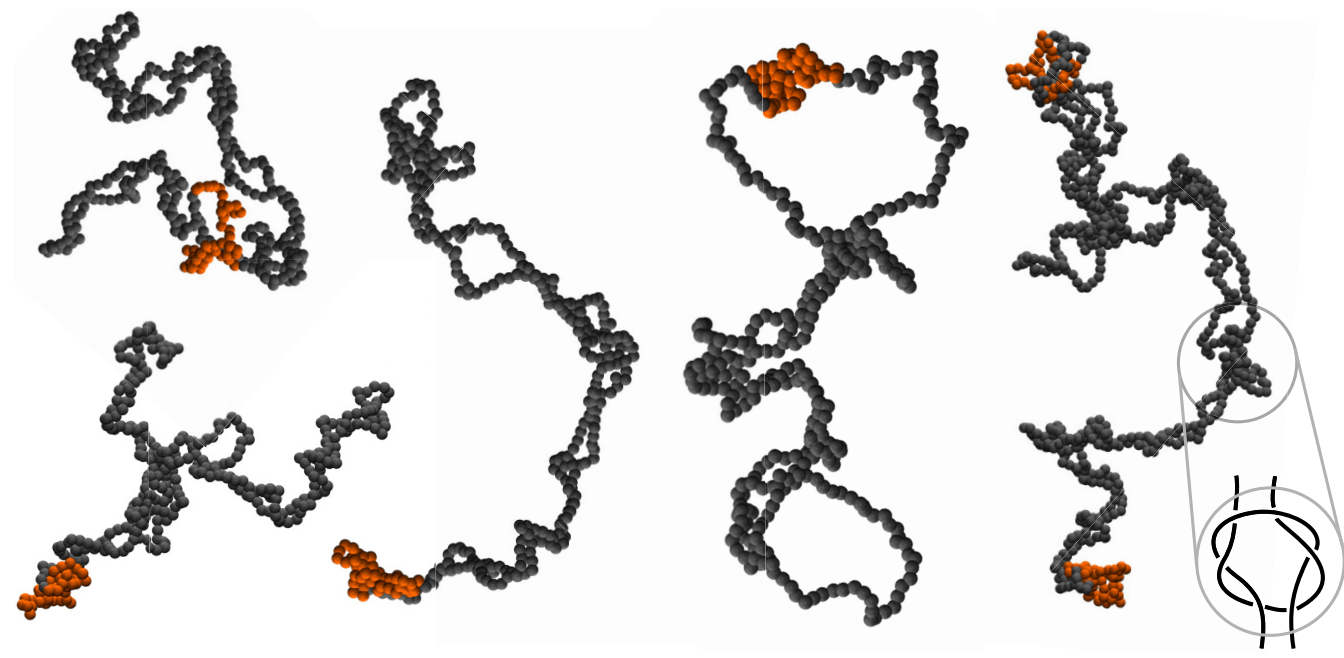

FIG. 1. Typical conformations of partially active rings in the glassy state. The shown configurations were randomly chosen from the ensemble of rings in the system with $N=400$. The orange monomers are active and the gray ones passive. Loopy openings on a ring highly likely correspond to other rings that thread it at a given place. In many cases, the conformations feature a doubly folded structure with the hot segment located at one of its ends. The rightmost configuration depicts two rings entangled by a tight threading similar to square knot structure schematically depicted in the inset.

activity in the latter case was induced by coupling a consecutive number of monomers on a ring to stronger thermal fluctuations than the rest, that is, by making them effectively hotter. The nonequilibrium dynamics of such partially active rings, stemming from a strongly broken detailed balance, accesses conformations unfavorable in equilibrium, dramatically increases inter-ring threading, and ensues substantial structural rearrangements of the system, ultimately resulting in a complete stall of the relative motion of the rings (typical ring conformations in the glassy state are shown in Fig. 1).

The relevance of such a complex nonequilibrium polymer system arises from its connection to the DNA fiber in living cells. On the DNA level, their life is supported by energy-dependent, active processes, such as DNA repair or transcription, that manifest themselves as stronger-thanthermal velocity fluctuations [25,26]. Although chromosomes of higher eukaryotes are typically linear, there exists a correspondence between their large-scale conformations and the typical conformations of ring polymers in a melt [15]. Additionally, circular DNA exists in bacteria, archaea, mitochondria, and chloroplast, but also eukaryotes possess so-called extra-chromosomal DNA that is circular and can be even highly transcriptionally active in some cancers [27]. While the biological aspect is certainly intriguing, here we are interested in the physical properties and mechanisms behind the formation of this state of matter.

Many properties of such nonequilibrium, activity-driven glassy states in ring polymer melts remain elusive, such as the relation between the glass transition and the active-passive phase separation. While pressure, temperature, and chemical potential are necessarily uniform across the system with different particle species in thermodynamic equilibrium, this is no longer the case for steady states of nonequilibrium active matter systems [28-31]. The active-passive phase separation is observed for various models of activity $[32,33]$ but coexistence conditions can depend on activity details [29].
In a two-temperature mixture, the degree of phase separation between two species strongly depends on the temperature difference between the hot and cold thermostats, the friction coefficient $\gamma$, which serves as a coupling constant between particles and thermostats, as well as on the polymerization degree of particles, provided they are polymers [30,31,3336]. In colloidal particle mixtures containing an equal number of fractions of both species, the phase segregation arises if the critical temperature ratio is quite high, $T_{\mathrm{h}} / T_{\mathrm{c}} \approx 30$ [33-35]. Such activity ratio roughly compares with the ratio of Péclet numbers necessary to observe phase separation of self-propelling active particles [32]. Nevertheless, as has been shown for the two-temperature case [30,31,35], the critical activity ratio decreases with the particles' polymerization degree $N$ with the incompatibility parameter scaling as $\sim N^{-1 / 2}$ [30,35], in contrast to $\sim N^{-1}$ dependence for equilibrium phase separation [37]. While the theoretical result [35] is built on a virial-like approximation and hence applicable to moderate densities, the scaling $\sim N^{-1 / 2}$ had been conjectured based on simulation of concentrated active-passive solutions [30].

In active topological glass, the sufficiently long active blocks would tend to segregate, but their diffusivity that plays a role in the enhancement of threading decreases with $N$. Natural questions arise: How is the vitrification related to the observed active-passive phase separation for polymer mixtures? What is the role of the ring length for a fixed activity ratio? How are the conformations, dynamics, and phase-separated morphologies of such nonequilibrium, activity-driven rings related to those of equilibrium and what are their scaling properties? We tackle these questions by considering melts of ring polymers of varying length subject to comparable activity quench.

In this paper, we detail how such a nonequilibrium system of rings arrives at a dynamically arrested state. The onset of activity implies a discrepancy between the dynamics of hot and cold segments that, as we show, if strong enough, 
generates a slithering-snakelike motion of the active segment, pulling the rest of the chain that has a propensity to form tight threadings-topological constraints that are difficult to resolve. Some of these have the local form of square knots (see inset of Fig. 1) and are found also to be responsible for a strong increase in viscosity for ring melts under extensional flow $[38,39]$. Once a sufficiently high number of such constraints is formed, the cold part of a ring is essentially fixed in space, whereas the hot one intermittently continues diffusing through the system, causing a significant and rapid expansion of the rings. As a result, it leads to even stronger inter-ring tangling. In the final, deeply threaded state, the hot segments tend to phase segregate more, which reduces energy dissipation and increases their effective temperature, leading to further tightening of the constraints. At this steady state, the constraints cause the dynamics of each ring to lose the drift element. As we show, the aforementioned process of segregation between hot and cold segments comes to a halt due to the vitrification caused by the circular topology of the ring polymers. To investigate the possible morphologies of activity-induced phase separation, we also examine steady states of other polymer architectures that can arise from cutting the rings, by which we remove the long-lasting topological constraints. In particular, linear mixtures of unequal lengths and linear triblock copolymer systems exhibit microphase separation, however, to the extent and morphology different from both their equilibrium phase-separated counterparts and the active topological glass.

\section{MODEL}

Our systems consist of $M=1600$ monodisperse, semiflexible ring polymers of length $N(N=100,200,400)$. We employ a standard model for polymer melts [40], in which excluded-volume interactions between monomers are given by the purely repulsive Lennard-Jones potential,

$$
U_{\mathrm{LJ}}(r)=\left(4 \varepsilon\left[\left(\frac{\sigma}{r}\right)^{12}-\left(\frac{\sigma}{r}\right)^{6}\right]+\varepsilon\right) \theta\left(2^{1 / 6} \sigma-r\right),
$$

where $\theta(x)$ denotes the Heaviside step function, $\sigma$ is the diameter of each monomer having mass $m$, and $\epsilon$ sets the energy scale. The bonding potential between two neighboring monomers along the chain's contour is set by the finitely extensible nonlinear elastic potential,

$$
U_{\mathrm{FENE}}(r)=-\frac{1}{2} r_{\max }^{2} K \ln \left[1-\left(\frac{r}{r_{\max }}\right)^{2}\right],
$$

with $K=30 \varepsilon / \sigma^{2}$ and $r_{\max }=1.5 \sigma$. Finally, the bending potential is

$$
U_{\text {angle }}=k_{\theta}[1-\cos (\theta-\pi)]
$$

with $k_{\theta}=1.5 \varepsilon$. This choice of parameter prevents interchain crossings that would violate the initial nonconcatenation constraint. Moreover, it achieves small entanglement length $N_{e}=$ 28 , below which the chains are still approximately Gaussian.

In each system, successive $N_{\mathrm{h}}=N / 8$ monomers on every ring are made active $\left(N_{\mathrm{h}}=13,25,50\right.$ for $N=100,200,400$, respectively). This is achieved by subjecting them to stronger thermal-like fluctuations, as compared to the remaining $N_{\mathrm{c}}=$ $N-N_{\mathrm{h}}$ particles. Here and in what follows, active monomers are interchangeably called hot, whereas passive ones are cold. In practice, we couple both species to two distinct Langevin thermostats with $T_{\mathrm{h}}=3 \varepsilon\left(k_{\mathrm{B}}=1\right)$ for active monomers, and $T_{\mathrm{c}}=1 \varepsilon$ for passive ones, such that the equations of motions read as

$$
m \dot{\mathbf{v}}_{i}=-m \gamma \mathbf{v}_{i}+\boldsymbol{F}_{i}+\left(2 m \gamma T_{i}\right)^{1 / 2} \boldsymbol{\eta}_{i},
$$

where $\gamma$ is the coupling constant with the heat bath, $\boldsymbol{F}_{i}$ is the total conservative force on the monomer, the components of $\boldsymbol{\eta}_{i}$ satisfy $\left\langle\eta_{i, \alpha}(t) \eta_{j, \beta}\left(t^{\prime}\right)\right\rangle=\delta_{i j} \delta_{\alpha \beta} \delta\left(t-t^{\prime}\right)$, and $T_{i}$ is either $T_{\mathrm{h}}$ or $T_{\mathrm{c}}$. The same $\gamma=(2 / 3) \tau^{-1}$, where $\tau=\sigma(m / \varepsilon)^{1 / 2}$, is used for both thermostats. These choices of $\gamma$ and $T_{\mathrm{h}}$ are sufficient to drive nonequilibrium phase separation in active-passive mixtures of short $(N \gtrsim 20)$ polymers [30,31] but by far not strong enough to observe demixing of colloids $[33,34]$. All the Langevin dynamics simulations were performed at constant volume and density $\rho=0.85 \sigma^{-3}$ using the large-scale atomic/molecular massively parallel simulator (LAMMPS) engine [41]. The integration time step was set to $\Delta t=0.005 \tau$. Finally, as noted in the original work [24], relatively large system sizes with $M=1600$ polymers were needed to ensure that unphysical self-threadings of extremely elongated rings due to periodic boundary conditions do not occur.

\section{ONSET OF GLASSY DYNAMICS}

At time $t_{0}=0 \tau$, the activity on all rings in a properly equilibrated sample of $M$ chains is switched on. As shown below, the ensued dynamics differs across the three cases considered: Whereas for the larger rings with $N=200$ and 400 , it progressively slows down and eventually results in a halt of the relative motion of the rings, the dynamics of the system with $N=100$ is equilibriumlike. In what follows, we investigate the relaxation of the systems toward a steady state, as indicated by the ring dynamics, their conformation and threading properties, and the phase separation between the active and passive monomers, as well as the final system properties in such steady states.

To characterize in detail the rings' motion on all scales, we consider the mean-square displacements of active and passive monomers, $g_{1}^{\alpha}\left(t, t_{0}\right)$ ( $\alpha=$ either hot or cold $)$, their meansquare displacements with respect to the ring's center of mass, $g_{2}^{\alpha}\left(t, t_{0}\right)$, and the mean-square displacements of the center of mass of the whole ring, $g_{3}\left(t, t_{0}\right)$ :

$$
\begin{gathered}
g_{1}^{\alpha}\left(t, t_{0}\right)=\left[\mathbf{r}^{\alpha}\left(t_{0}+t\right)-\mathbf{r}^{\alpha}\left(t_{0}\right)\right]^{2}, \\
g_{2}^{\alpha}\left(t, t_{0}\right)=\left[\Delta \mathbf{r}^{\alpha}\left(t_{0}+t\right)-\Delta \mathbf{r}^{\alpha}\left(t_{0}\right)\right]^{2}, \\
g_{3}\left(t, t_{0}\right)=\left[\mathbf{R}\left(t_{0}+t\right)-\mathbf{R}\left(t_{0}\right)\right]^{2},
\end{gathered}
$$

where $\mathbf{r}^{\alpha}(t)$ is the position of an active or passive monomer at time $t, \mathbf{R}$ is the center of mass position of the ring, and $\Delta \mathbf{r}^{\alpha}(t)=\mathbf{r}^{\alpha}(t)-\mathbf{R}(t)$ is the position of a monomer with respect to its ring's center of mass. In all cases, the positions are relative to the total center of mass of the system at the given time to subtract the induced global drift. Additionally, we consider the mean-square displacements of the center of mass of active and passive segments, $g_{3}^{\alpha}\left(t, t_{0}\right)$ :

$$
g_{3}^{\alpha}\left(t, t_{0}\right)=\left[\mathbf{R}^{\alpha}\left(t_{0}+t\right)-\mathbf{R}^{\alpha}\left(t_{0}\right)\right]^{2},
$$



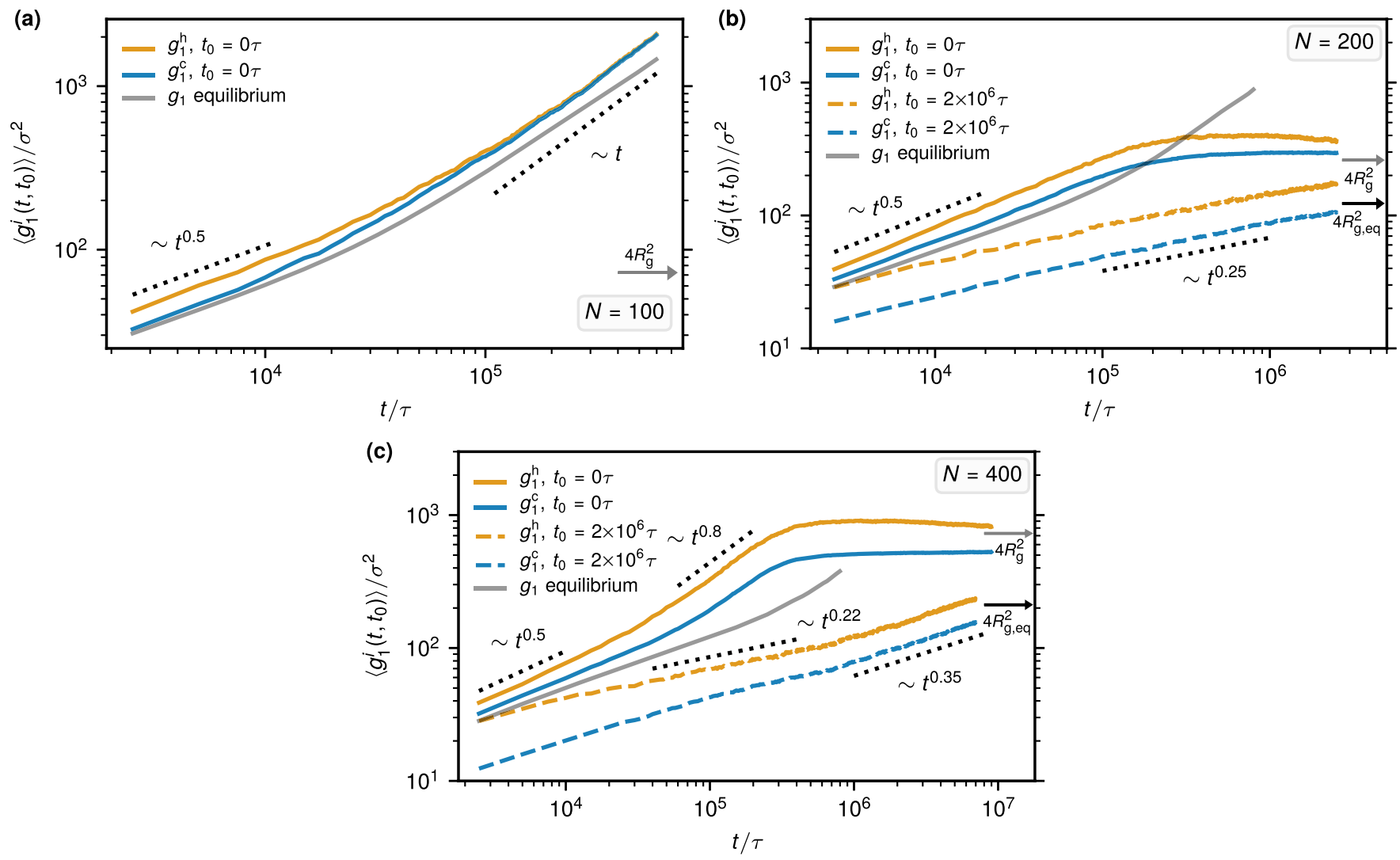

FIG. 2. Dynamics of monomers. Mean-square displacements of active, $g_{1}^{\mathrm{h}}\left(t, t_{0}\right)$, and passive, $g_{1}^{\mathrm{c}}\left(t, t_{0}\right)$, monomers in the early $\left(t_{0}=0 \tau\right.$, solid lines) and late ( $t_{0}=2 \times 10^{6} \tau$, dashed lines) stages for systems with $N=100$ (a), $N=200$ (b), and $N=400$ (c). The partially active rings with $N=100$ feature equilibriumlike dynamics that does not depend on $t_{0}$, thus only the data for $t_{0}=0 \tau$ is shown in (a). The dashed lines indicate intermediate scaling regimes. The transparent gray lines correspond to $g_{1}$ in equilibrium for the respective case. The arrows indicate the squared equilibrium (black arrows) and nonequilibrium (gray arrows) diameter of gyration.

where $\mathbf{R}^{\alpha}(t)=N_{\alpha}^{-1} \sum_{i=1}^{N_{\alpha}} \mathbf{r}_{i}^{\alpha}(t)$ is the center-of-mass position of the active or passive segment on a ring. In general, we consider only the mean-square displacements averaged over rings, $\left\langle g_{i}^{\alpha}\left(t, t_{0}\right)\right\rangle$, without additional averaging over multiple time origins because of the explicit dependence of the dynamics on $t_{0}$ at early times. The steady-state properties for the systems with $N=200$ and 400 correspond to the time when most conformational properties come to a standstill and to when the dynamic correlation functions do not feature a significant dependence on $t_{0}$. For the former two systems, this time corresponds to about $t_{0} \simeq 2 \times 10^{6} \tau$. Thus, in what follows we will focus on two cases: early rearrangements immediately after the activity onset $\left(t_{0}=0 \tau\right)$ as well as the steady-state dynamics $\left(t_{0}=2 \times 10^{6} \tau\right)$. Finally, to give a perspective on the dynamics, we also simulated fully equilibrium systems with $T_{\mathrm{h}}=T_{\mathrm{c}}=1$ and all other parameters the same as in the active cases.

As mentioned in the Introduction, the friction coefficient $\gamma$ is an important parameter that governs redistribution of heat between the cold and hot subsystems. In Langevin dynamics simulations, lower values of $\gamma$ enhance the heat transfer between distinct particle species and therefore result in apparent effective temperatures $T_{\mathrm{h}}^{\text {eff }}$ and $T_{\mathrm{c}}^{\text {eff }}$ of hot and cold particles that differ from the ones set by the thermostats $[30,31,36]$ :

$$
T_{\mathrm{c}}<T_{\mathrm{c}}^{\mathrm{eff}}<T_{\mathrm{h}}^{\mathrm{eff}}<T_{\mathrm{h}} .
$$

Conversely, the increase of $\gamma$ keeps the particles' temperatures closer to the thermostat ones, thereby amplifying the effective temperature difference and thus promoting phase segregation of hot and cold particles. Finally, due to the heat flow to the cold subsystem, even the diffusivity of passive monomers is increased because their effective temperature is higher than the one imposed by the thermostat, $T_{\mathrm{c}}^{\mathrm{eff}}>T_{\mathrm{c}}$. In any case, we use thermostat coupling constant much lower than the effective bead-bead friction $\left(\simeq 20 \tau^{-1}\right)$ [42].

As shown in Fig. 2, the early dynamics $\left(t_{0}=0 \tau\right)$ of both active and passive monomers is consistent with that of equilibrium melts $[3,4,43]$, and is characterized by $g_{1}^{\mathrm{h}(\mathrm{c})}$ scaling as $g_{1}^{\mathrm{h}(\mathrm{c})} \sim t^{1 / 2}$, indicating standard Rouse behavior. For all $N$, in full accordance with Eq. (9), at early times $g_{1}^{\mathrm{h}}>g_{1}^{\mathrm{c}}>g_{1}$, where $g_{1}$ corresponds to a completely passive, equilibrium system with $T_{\mathrm{h}}=T_{\mathrm{c}}=1$. In sharp contrast to $N=200$ and $N=400$, the $g_{1}^{\mathrm{h}(\mathrm{c})}$ for the system with $N=100$ crosses over to free diffusion $g_{1}^{\mathrm{h}(\mathrm{c})} \sim t$ with no indications of glassy behavior [Fig. 2(a)]. Moreover, the dynamics of the system with $N=100$ is equilibriumlike and does not depend on $t_{0}$. In contrast, the Rouse regime of $g_{1}^{\mathrm{h}(\mathrm{c})}$ for the two larger systems with $N=200$ and 400 is followed by a gradual slowdown, see Fig. 3 for time traces at early and late stages of the evolution. The steady-state dynamics of $g_{1}^{\mathrm{h}(\mathrm{c})}$ for active and passive monomers in these systems $\left(t_{0}=2 \times 10^{6} \tau\right)$ is indicated by 
(a)

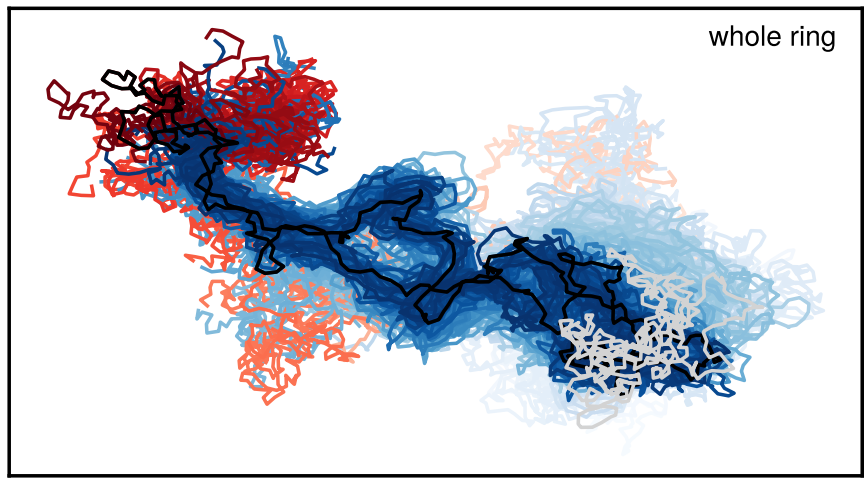

(b)

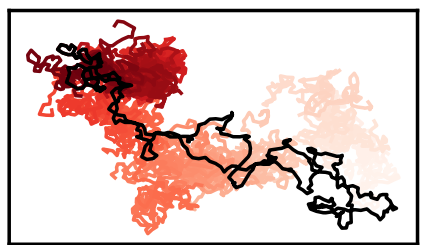

(c) passive segment

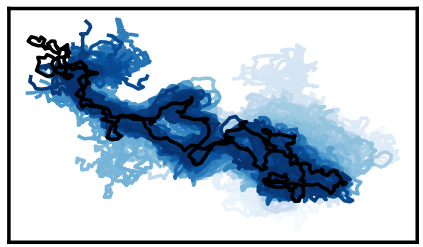

(d) Late evolution

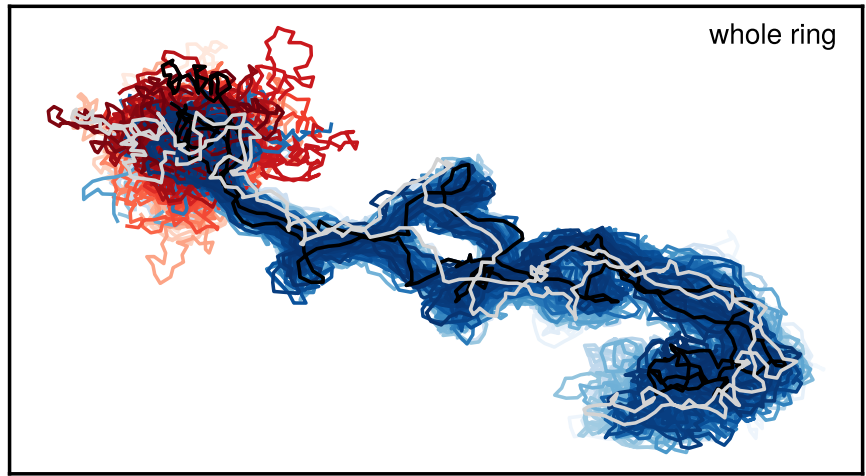

(e)

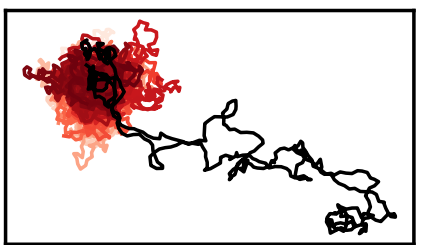

(f) passive segment

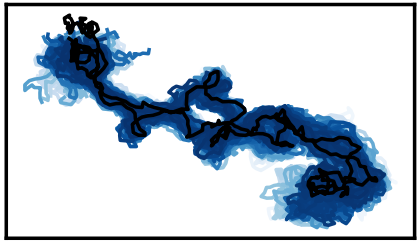

FIG. 3. Evolution of a single ring with $N=400$. Left panel: Early rearrangements of the conformation of the whole ring (a), its hot (b) and cold (c) segment over $t=5 \times 10^{5} \tau$ starting from the activity onset at $t_{0}=0 \tau$. Right panel: Late rearrangements of the conformation of the whole ring (d), its hot (e) and cold (f) segment over the subsequent $t=5 \times 10^{6} \tau$. In all plots, the black curve indicates the final ring configuration, whereas in (a) and (d), the light gray curve in the foreground indicates its starting configuration. In all cases, the $x y$ projection of the ring's coordinates is shown and the opacity increases linearly with time, that is, earlier configurations are lighter. The time trace of the hot and cold segments are shown in red and blue, respectively. Finally, note that the length of the shown trajectory in (d)-(f) is ten times longer than in (a)-(c).

dashed lines in Fig. 2: For short lag times, $g_{1}^{\mathrm{h}(\mathrm{c})}$ are generally slower than in the equivalent equilibrium case (especially, for passive monomers), $g_{1}^{\mathrm{h}}$ is typically $1.5-2$ times higher than $g_{1}^{\text {c }}$, and both feature a more than two-decade-long regime with $g_{1}^{\mathrm{h}(\mathrm{c})}$ exhibiting scaling close to $\sim t^{1 / 4}$. The exponent reminisces of linear polymer reptation along its chain contour [4]. In the present case, in the steady state the centers of mass of the rings are practically fixed due to numerous threadings, and their displacements are only generated by occasional pulls of the active segments, as we detail below in Fig. 4. This essentially corresponds to a directed reptationlike motion where the active segment serves as an effective "chain end" that directs ring's displacements.

For the two larger systems $(N=200,400)$ that slow down, in Fig. 4 we further compare the mean-square displacements of the center of mass of active and passive segments, $g_{3}^{\mathrm{h}(\mathrm{c})}$, as well as of the whole ring, $g_{3}$, in the early $\left(t_{0}=0 \tau\right)$ and late $\left(t_{0}=2 \times 10^{6} \tau\right)$ stages. Similarly to $g_{1}^{\mathrm{h}(\mathrm{c})}$, the early dynamics
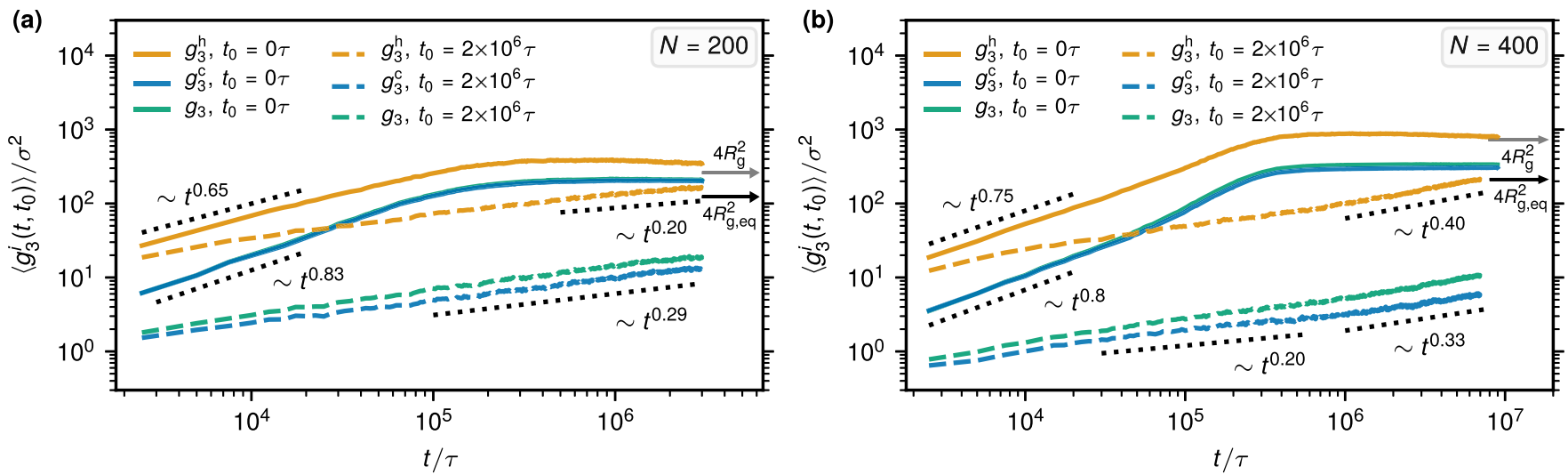

FIG. 4. Dynamics of segments. Comparison between the center-of-mass mean-square displacements of active, $g_{3}^{\mathrm{h}}\left(t, t_{0}\right)$, and passive, $g_{3}^{\mathrm{c}}\left(t, t_{0}\right)$, segments, and the whole rings, $g_{3}\left(t, t_{0}\right)$, for $N=200$ (a) and 400 (b) in the early $\left(t_{0}=0 \tau\right.$, solid lines) and late $\left(t_{0}=2 \times 10^{6} \tau\right.$, dashed lines) stages. The dotted lines indicate different intermediate scaling regimes. The arrows indicate the squared equilibrium (black arrows) and nonequilibrium (gray arrows) diameter of gyration. 

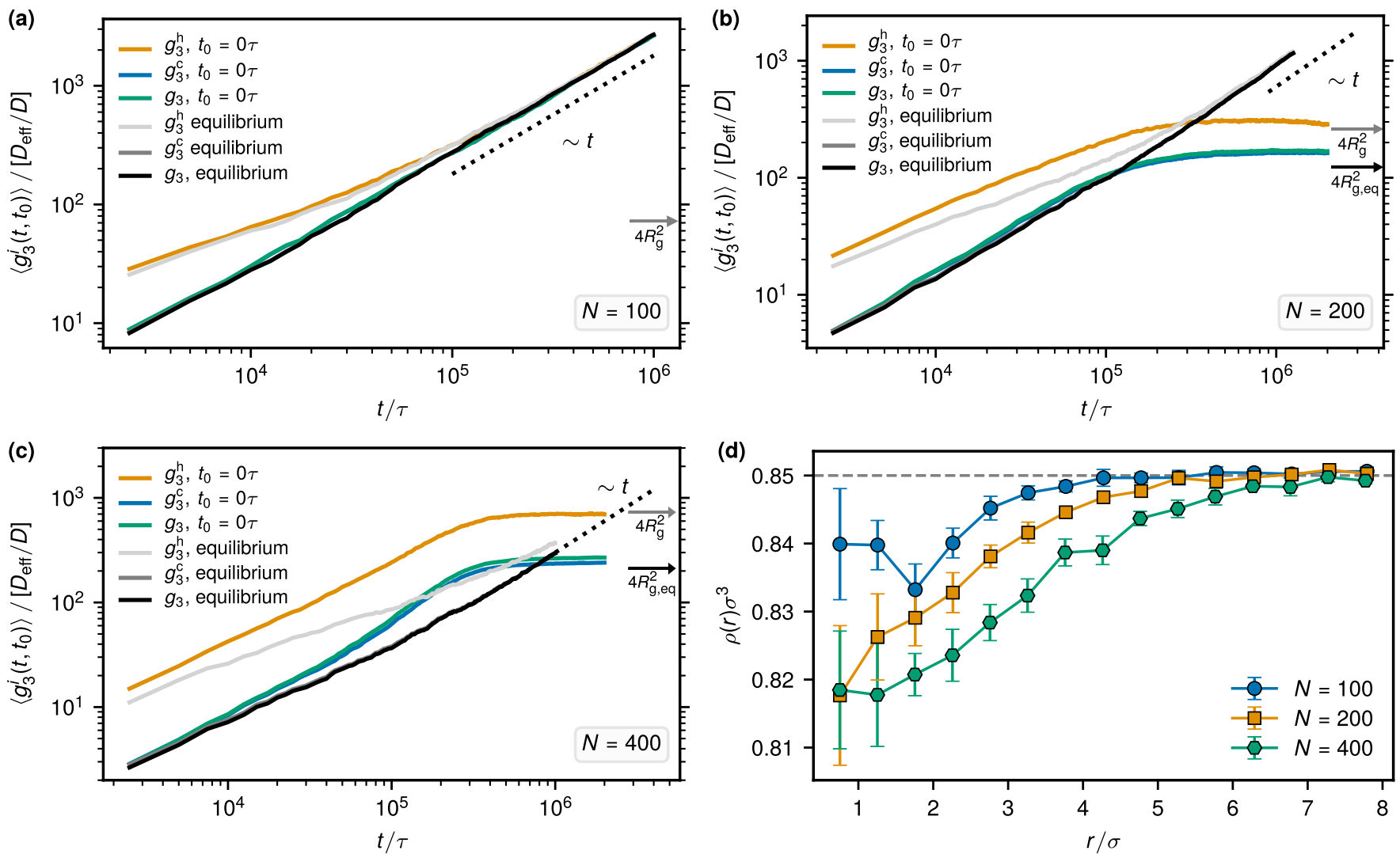

FIG. 5. Dynamics of segments rescaled with effective diffusion coefficient. Comparison between the center-of-mass mean-square displacements of active, $g_{3}^{\mathrm{h}}\left(t, t_{0}\right)$, and passive, $g_{3}^{\mathrm{c}}\left(t, t_{0}\right)$, segments, and the whole rings, $g_{3}\left(t, t_{0}\right)$, for $N=100$ (a), $N=200$ (b), and 400 (c) in the early $\left(t_{0}=0 \tau\right)$ stage $\left(D_{\text {eff }} / D=1.25\right)$ and the equivalent dependencies in equilibrium $\left(D_{\text {eff }} / D=1\right)$. The mean-squared gyration radius in equilibrium $R_{\mathrm{g}, \mathrm{eq}}^{2}$ [3] and in the steady-state $R_{\mathrm{g}}^{2}$ are marked by arrows for comparison to the mean-squared displacements. (d) Average monomer density around the center of mass of the hot segment early after the activity onset $\left(t<10^{4} \tau\right)$. For all $N$, the dip in the density has width of about one diameter of gyration of the hot segment at that time. The gray dashed line corresponds to the mean monomer density $\rho \sigma^{3}=0.85$.

of $g_{3}^{\mathrm{h}(\mathrm{c})}$ and $g_{3}$ is consistent with the short-time dynamics of an equilibrium ring melt with $g_{3} \sim t^{3 / 4}$ [4]. Naturally, due to a significant difference in length, the hot segment at early times diffuses faster than the cold one, as evidenced by about an order of magnitude difference between $g_{3}^{\mathrm{h}}$ and $g_{3}^{\mathrm{c}}$. However, in the present cases, the hot segment still diffuses faster than it would be in a corresponding equilibriumlike model. As shown in Appendix A, the center of mass of a free polymer chain, parts of which are connected to two distinct thermostats, follows equilibriumlike dynamics with an effective temperature, $T_{\text {eff }}$, which is a weighted average of the two thermostat temperatures $T_{\text {eff }}=\left(T_{\mathrm{c}} N_{\mathrm{c}}+T_{\mathrm{h}} N_{\mathrm{h}}\right) / N$, directly translating into its enhanced diffusion coefficient $D_{\text {eff }} \sim T_{\text {eff }}$. Although the above relations are given in terms of the thermostat temperatures $T_{\mathrm{h}(\mathrm{c})}$, the same $T_{\mathrm{eff}}$ is obtained if $T_{\mathrm{h}(\mathrm{c})}$ are replaced with $T_{\mathrm{h}(\mathrm{c})}^{\mathrm{eff}}$, which is due to the balance of heat fluxes that is discussed later on in Sec. V. For all systems considered here, $N_{\mathrm{h}}=N / 8$ and $T_{\mathrm{h}}=3 T_{\mathrm{c}}$, therefore $D_{\text {eff }}=1.25 D$ with $D$ being the equilibrium diffusion coefficient $\left(T_{\mathrm{h}}=T_{\mathrm{c}}\right)$. The Rouse regime is well applicable until the chain starts to explore topological constraints imposed by other chains. For shorter rings, these do not present a significant obstacle, as their (half) length is only moderately higher than the equilibrium entanglement length, which itself is effectively higher for hotter melt due to enhanced chain flexibility [44].
In Fig. 5, we show $g_{3}^{\mathrm{h}(\mathrm{c})}$ as well as $g_{3}$ rescaled by $D_{\text {eff }} / D$ and compare them to the equivalent equilibrium cases for all $N$. While we find a good correspondence between the rescaled displacements for $N=100$, for $N=200$ and $N=400$ certain differences arise already at early times, immediately after the activity onset. In particular, the dynamics of the cold segment and the center of mass are generally consistent with the equilibriumlike system with enhanced $D_{\text {eff }}$ at early times, whereas the hot segment still diffuses faster as compared to the latter case. This arises from a more dilute environment around hot segments [see Fig. 5(d)] that indicates an early stage of nonequilibrium phase segregation. The active segments not only feature a higher diffusivity because of their coupling to a hotter heat bath but also because of a lower density around them. In equilibrium, such a moderate decrease in density has a pronounced effect on the effective monomer friction and the resulting diffusion coefficient [42]. As seen in Fig. 4, the discrepancy between the segment dynamics in the two larger systems only deepens in the steady state, which is consistent with further demixing of hot and cold monomers, as detailed later on in Sec. V. Similarly to the monomer dynamics, centerof-mass mean-square displacements $g_{3}^{\mathrm{h}(\mathrm{c})}$, the quantity $g_{3}$ in the steady state $\left(t_{0}=2 \times 10^{6} \tau\right)$ features a very slow relaxation that scales as $\sim t^{0.2}$ at shorter times and then appears to cross over to $\sim t^{0.3-0.4}$ at longer ones. Note that in the steady 


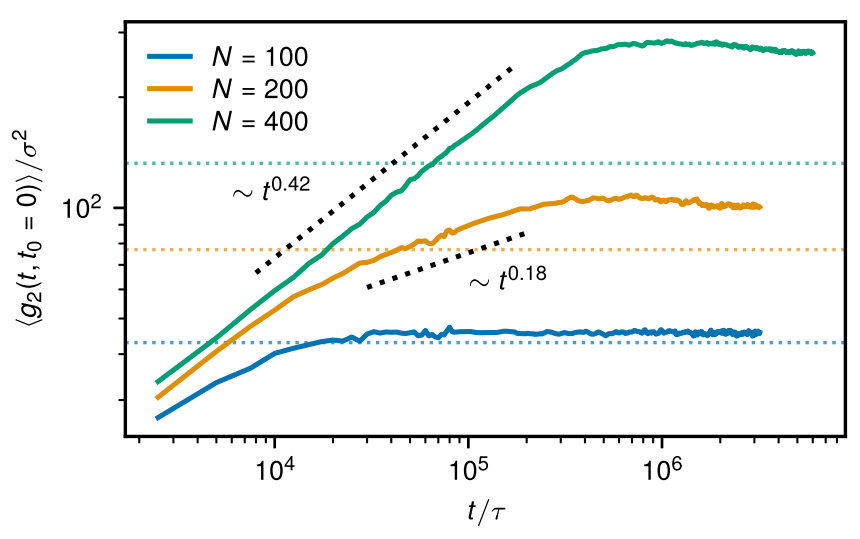

FIG. 6. Dynamics of monomers with respect to the center of mass. $g_{2}\left(t, t_{0}\right)$ is shown immediately after the onset of activity, that is, for $t_{0}=0 \tau$, for systems with different $N$ and is averaged over both hot and cold monomers in the system. There is practically no difference in $g_{2}^{\mathrm{c} / \mathrm{h}}$ computed separately for cold and hot monomers. The black dotted lines indicate intermediate scaling regimes. The colored dotted lines indicate the value $2.5\left\langle R_{\mathrm{g}}^{2}\right\rangle$, where $\left\langle R_{\mathrm{g}}^{2}\right\rangle$ is the squared equilibrium radius of gyration taken from [3] (different colors corresponds to different $N$ as in the legend). After around $2.5\left\langle R_{\mathrm{g}}^{2}\right\rangle, g_{3}$ of an equilibriumlike system would cross over to diffusion, whereas $g_{2}$ would saturate (see more discussion in the main text).

state, the displacement of the total center of mass is very small ( $\simeq 3 \sigma$ for time $\simeq 10^{7} \tau$ ), while the hot segment explores distances about $10 \sigma$ [Fig. 4(b)]. As the number of active monomers is relatively small compared to the length of the whole ring, the center-of-mass mean-square displacements, $g_{3}$, generally resemble those of the passive segment. This further confirms the picture that the active segments explores the neighboring environment, while the whole ring remains essentially fixed due to pronounced topological constraints.

In equilibrium ring melts, the center of mass of rings of these still relatively short lengths starts to diffuse after traveling about $2\left\langle R_{\mathrm{g}}^{2}\right\rangle\left(\left\langle R_{\mathrm{g}}^{2}\right\rangle\right.$ is the mean-square ring's radius of gyration), which corresponds to the point where $g_{3}$ and $g_{2}$ cross [4]. In the present nonequilibrium case with $N=100$, we find that this happens at around $2\left\langle R_{\mathrm{g}}^{2}\right\rangle D_{\text {eff }} / D=$ $2.5\left\langle R_{\mathrm{g}}^{2}\right\rangle$, with $\left\langle R_{\mathrm{g}}^{2}\right\rangle$ being the equilibrium value. Figure 6 shows $\left\langle g_{2}\left(t, t_{0}\right)\right\rangle$ immediately after the activity onset $\left(t_{0}=\right.$ $0 \tau)$ and averaged over both active and passive monomers ( $g_{2}$ computed separately for the two species are practically indistinguishable). While for $N=100, g_{2}$ indeed saturates after about $2.5\left\langle R_{\mathrm{g}}^{2}\right\rangle$, this is not the case for the two longer polymers. In the latter cases, $g_{2}$ grows beyond $2.5\left\langle R_{\mathrm{g}}^{2}\right\rangle$, which is an indication of the rings expansion past the equilibrium size. In all cases, at short times, $g_{2}$ features scaling close to the Rouse-like regime of $g_{1}, g_{2} \sim t^{1 / 2}$. For $N=400$, such regime governs $g_{2}$ up to the point where it begins to saturate. For $N=200, g_{2} \sim t^{1 / 2}$ scaling crosses over to $g_{2} \sim t^{0.2}$ before saturation. As will be seen in Sec. IV, this corresponds to slower expansion of ring's size for $N=200$, as compared to $N=400$.

In Fig. 7, we further compare the mean-square displacements of the center of mass of the rings for systems with different $N$. We find that the time of the onset of the slowdown of the dynamics for $N=200$ and $N=400$ roughly

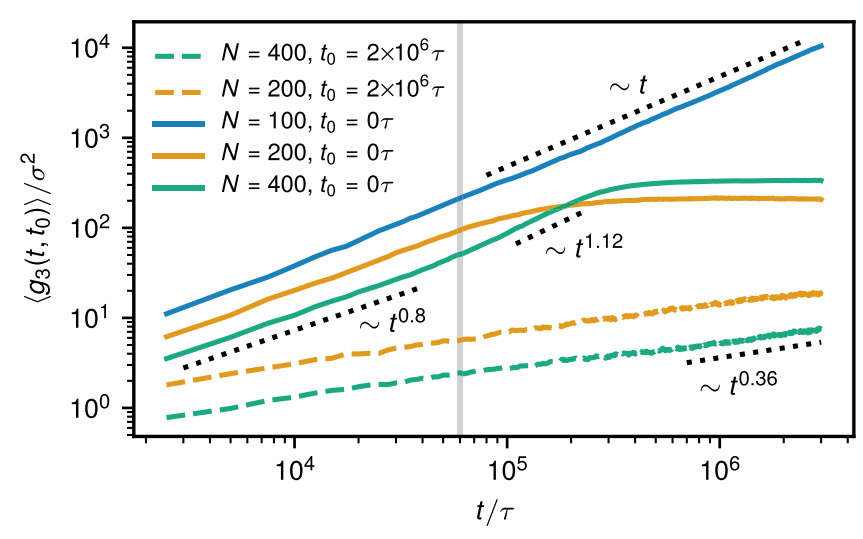

FIG. 7. Dynamics of the center of mass. Mean-square displacements of the ring's center of mass in the early $\left(t_{0}=0 \tau\right.$, solid lines) and late $\left(t_{0}=2 \times 10^{6} \tau\right.$, dashed lines) stages for systems with different $N$. Partially active rings with $N=100$ feature equilibriumlike dynamics that does not depend on $t_{0}$. The dotted lines indicate intermediate scaling regimes. The gray line indicates the time $t=$ $6 \times 10^{4} \tau$. (see also Fig. 8).

corresponds to the onset of free diffusion in equivalent equilibrium ring melts [4]. Conversely, $N=100$ crosses over from the $g_{3}$ exponent of about 0.8 to standard diffusion with the corresponding diffusion coefficient being about $25 \%$ higher than in equilibrium, in full agreement with considerations in Appendix A and Eq. (A3), giving $D_{\text {eff }}=1.25 D$. While both $N=200$ and $N=400$ arrive at an arrested state, the exact mechanism of how they do that differs in these two cases. In particular, the Rouse regime for $g_{1}^{\mathrm{h}(\mathrm{c})}$ in the system with $N=200$ extends beyond the crossover to diffusion in the equivalent equilibrium system [Fig. 2(b)], whereas $g_{1}^{\mathrm{h}(\mathrm{c})}$ in the system with $N=400$ is crossing over toward diffusion earlier than in equilibrium, however, saturates later on [Fig. 2(c)]. For $N=400$, the crossover to diffusion of $g_{1}^{\mathrm{h}(\mathrm{c})}$ coalesces with super-diffusive motion of the center of mass (Fig. 7), during which the rings expand significantly (see also a detailed discussion on the present superdiffusion mechanism in Ref. [24]). For $N=200$, such a superdiffusive regime is not observed and it is likely caused by the shorter ring length that limits the amount by which the rings can expand while being driven by the hot segment.

\section{CONFORMATIONAL PROPERTIES}

In Fig. 8, we quantify conformational rearrangements of the rings by tracking the time evolution of the mean radius of gyration,

$$
R_{\mathrm{g}} \equiv\left\langle R_{\mathrm{g}}^{2}\right\rangle^{1 / 2}=\left\langle\frac{1}{N} \sum_{i=1}^{N}\left(\mathbf{r}_{i}-\mathbf{R}\right)^{2}\right\rangle^{1 / 2},
$$

and the mean "end-to-end" distance,

$$
R_{\mathrm{e}} \equiv\left\langle R_{\mathrm{e}}^{2}\right\rangle^{1 / 2}=\left\langle\frac{1}{N} \sum_{i=1}^{N}\left(\mathbf{r}_{i+N / 2}-\mathbf{r}_{i}\right)^{2}\right\rangle^{1 / 2},
$$

where, $\mathbf{r}_{i}$ denotes the position of the $i$ th monomer and $\mathbf{R}$ is the position of the center of mass of the ring. Additionally, 

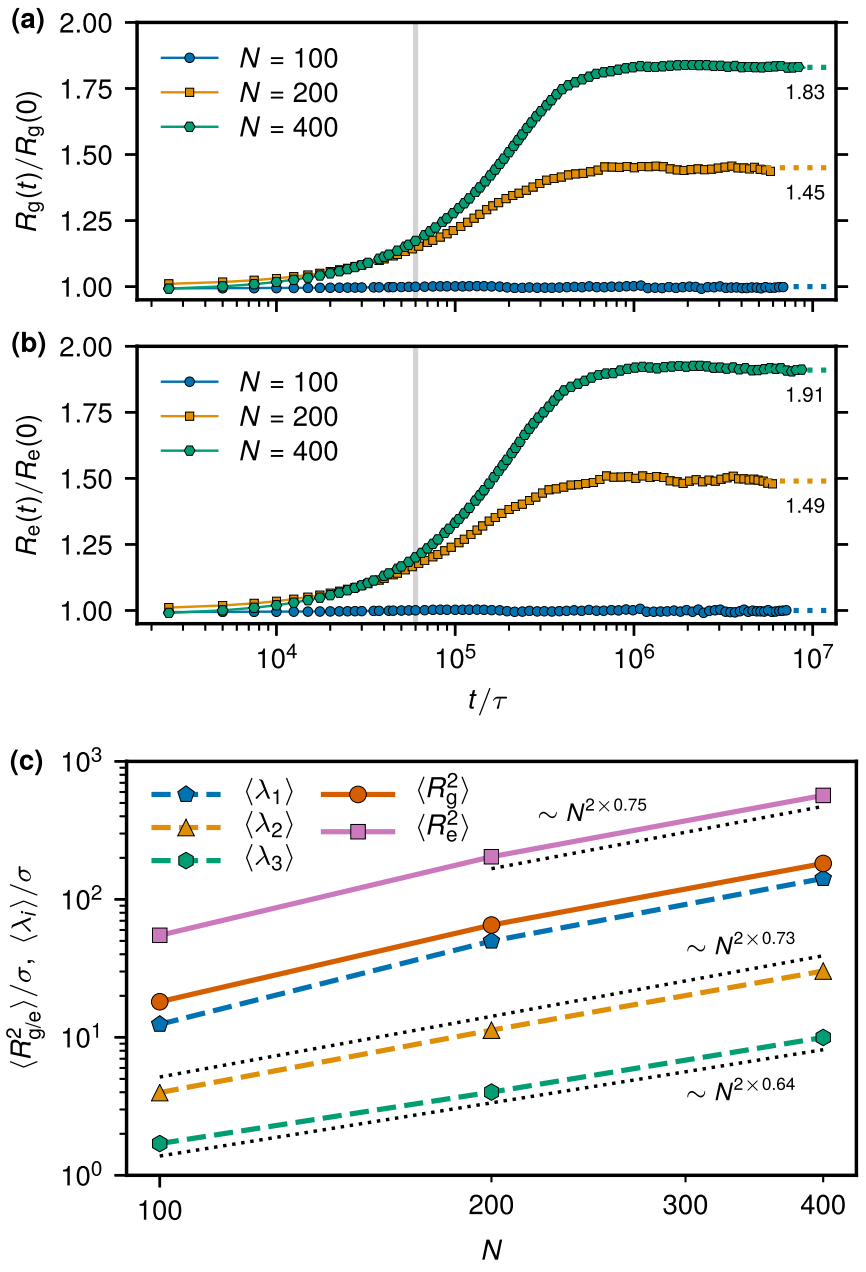

FIG. 8. Size properties. Time evolution of (a), the mean radius of gyration, $R_{\mathrm{g}}$, and (b) the mean "end-to-end" distance, $R_{\mathrm{e}}$, of the rings. The time series of $R_{\mathrm{g}}(t)$ and $R_{\mathrm{e}}(t)$ are scaled with their values at $t=0 \tau$ that correspond to equilibrium conformations. The gray lines in (a) and (b) indicate the time $t=6 \times 10^{4} \tau$, at which significant slowdown of rings' dynamics in the two larger systems becomes apparent (see Fig. 7). Scaling of different size descriptors with $N$ in the steady state $\left(t>2 \times 10^{6} \tau\right)$ is shown in (c).

to quantify shape properties, we computed eigenvalues $\lambda_{i}$ ( $i=1,2,3, \lambda_{1} \geqslant \lambda_{2} \geqslant \lambda_{3}$ ) of the ring's gyration tensor $G_{i j}=$ $N^{-1} \sum_{k=1}^{N} \Delta r_{i}^{(k)} \Delta r_{j}^{(k)}$, where $\Delta r_{i}^{(k)}$ is the $i$ th component of the $k$ th monomer's position vector in its ring's center of mass frame. The angles $\langle\cdots\rangle$ stand for the ensemble average, which is generally time dependent due to the nonequilibrium character, of the systems over the the different rings at a given time. Whereas the system with $N=100$ that does not vitrify features almost the same $R_{\mathrm{g}}$ and $R_{\mathrm{e}}$ as in equilibrium, the systems with $N=200$ and 400 are characterized by a considerable growth of these quantities (steady-state size and shape parameters are given in Table I). The growth of the rings' size is associated to two effects. First, the change in the dynamics to oriented reptationlike impacts the ring shape that has to adapt to the topological constraints and establish a doubly folded configuration. Second, the formation of unresolvable threadings, which essentially fix parts of the rings, and the following pulling of the active segment extends the
TABLE I. Size and shape properties of the partially active rings at the steady state. $\left\langle R_{\mathrm{g}}^{2}\right\rangle$ is the mean-square radius of gyration, $\left\langle R_{\mathrm{e}}^{2}\right\rangle$ is the mean-square distance between two monomers separated by the contour length $N / 2$, and $\lambda_{i}, i=1,2,3$ are the eigenvalues of the gyration tensor ordered such that $\lambda_{1} \geqslant \lambda_{2} \geqslant \lambda_{3}$. The value in the parentheses indicates the standard error. For comparison with equilibrium values, please see Table II in Appendix B.

\begin{tabular}{rrrrrr}
\hline \hline$N$ & $N_{\mathrm{h}}$ & \multicolumn{1}{c}{$\left\langle R_{\mathrm{g}}^{2}\right\rangle / \sigma^{2}$} & \multicolumn{1}{c}{$\left\langle R_{\mathrm{e}}^{2}\right\rangle / \sigma^{2}$} & $\left\langle\lambda_{1}\right\rangle /\left\langle\lambda_{3}\right\rangle$ & $\left\langle\lambda_{2}\right\rangle /\left\langle\lambda_{3}\right\rangle$ \\
\hline 100 & 13 & $18.1(0.1)$ & $54.9(0.1)$ & $7.3(0.1)$ & $2.34(0.01)$ \\
200 & 25 & $65.2(0.3)$ & $203.5(3.8)$ & $12.4(0.1)$ & $2.81(0.01)$ \\
400 & 50 & $182.1(0.7)$ & $566.1(2.1)$ & $14.2(0.2)$ & $3.03(0.02)$ \\
\hline \hline
\end{tabular}

chain. Whether the unresolvable threadings are present from the initial stages or they appear only after the chain moves significantly is an open question. Currently, we favor the former scenario, as the rings move only about their own size. Based on observations of systems with fewer active chains where the vitrification appears later and passive chains are stretched, we think both scenarios are possible. Finally, the relaxation of $R_{\mathrm{g}}$ in the nonergodic steady state is markedly different as compared to equilibrium ring polymer melts (see Fig. S2 in Ref. [45]).

Such dramatic increase in the rings' size is associated with a similar increase in the number of rings' neighbors, $K_{1}(a)$, as shown in Fig. 9 (two rings are considered to be neighbors if their centers of mass lie at a distance smaller than $a$ ). Interestingly, a significant slowdown of the dynamics occurs after the rings on average grow by 10\% (see Figs. 7 and 8, and note the associated time scale highlighted by the gray lines). This further confirms that the heterogeneous dynamics of the rings' segments first promotes the formation of topological constraints, which eventually cause the vitrification of the system. Just before the dynamics significantly slows down, the
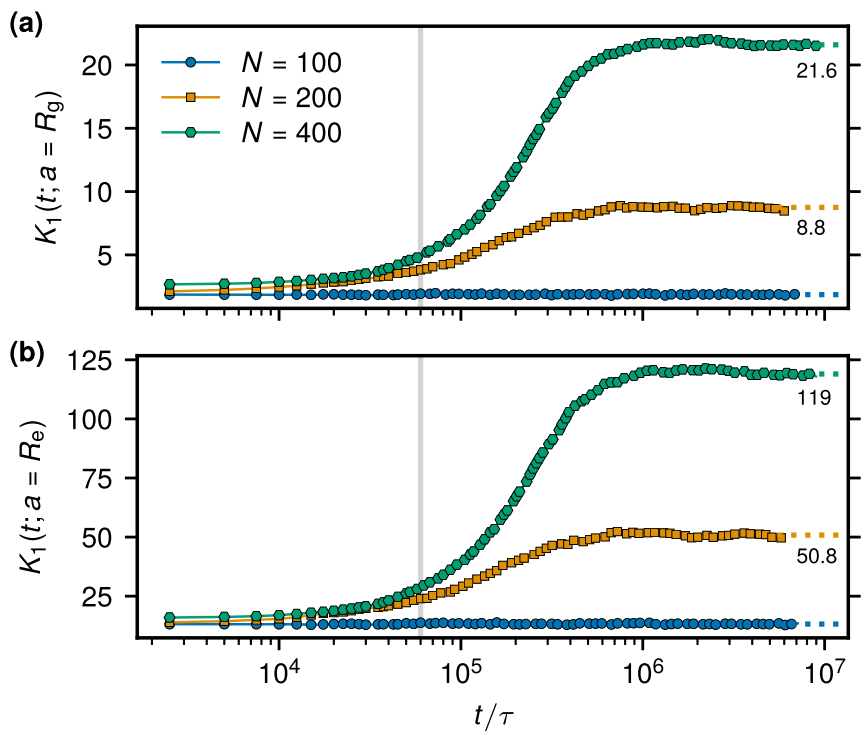

FIG. 9. The mean number of neighbors. Time evolution of the mean number of neighbors $K_{1}(t ; a)$ for(a) $a=R_{\mathrm{g}}$ and (b) $a=R_{\mathrm{e}}$. The gray line indicates the timescale at which significant slowdown of rings' dynamics in the two larger systems becomes apparent. 


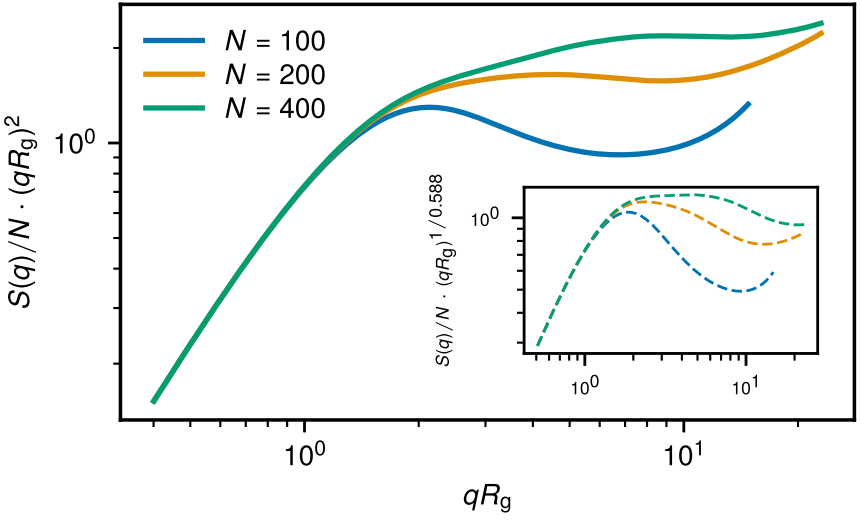

FIG. 10. Single chain static structure factor. $S(q)$ for different $N$ is multiplied by $q^{1 / v}$ with $v=1 / 2$ characterizing linear polymer chains. Inset: $S(q) q^{1 / v}$ but with $v=0.588$.

systems with $N=200$ and $N=400$ feature a limited regime of their size growth. This translates into an equivalent simultaneous growth of the average number of neighbors (Fig. 9). For compact objects, $K_{1}(a)$ is independent of $N$ while, for equilibrium, melt of linear chains it is proportional to $N^{1 / 2}$. Here, in the steady state, $K_{1}\left(R_{\mathrm{g}}\right)$ grows superlinearly, indicating that the stretching of the rings is stronger the longer the ring is. Additionally, as shown in Fig. 8, we quantified the scaling of different size parameters $\left(R_{\mathrm{g}}, R_{\mathrm{e}}, \lambda_{i}\right)$ with $N$, resulting in an apparent scaling $\sim N^{v}$ with $v$ taking a value $0.64-0.75$, distinctly above $1 / 2$, characterizing linear polymers in melt and $2 / 5$ and $1 / 3$ that describe rings of similar length [3]. Although this scaling is extracted from very few points only and can be attributed to a crossover between two distinct scaling regimes, the effective exponent higher than $1 / 2$ could be related to results on transversal fluctuations of directed polymers [46]. Similarly to directed polymers, present rings are being driven by the hot segment, although in random directions, and move trough a "gel matrix" formed by other rings. However, at these chain lengths $N$, we are not in the asymptotic limit and, therefore, not only the ring conformations differ slightly for different $N$, as shown below, but also the gel matrix is different. Therefore, a proper connection between the directed polymers and the present system is yet to be explored. Finally, the rings in the two bigger systems arrive at significantly stretched and elongated conformations with their radius of gyration and eigenvalue ratios being comparable to linear polymer chains of the same length [3], but their $R_{\mathrm{e}}$ comparable to the linear chains of half the length (compare Table I to Table II in Appendix B). This highlights that the rings are mostly doubly folded and significantly stretched in the vitrified state in comparison to equilibrium.

To gain a better understanding of conformations of such nonequilibrium rings, we evaluated the single chain structure factor $S(q)$, which is shown in Fig. 10. For a fractal object with dimension $1 / v, S(q)$ shall feature a scaling regime $S(q) \sim$ $q^{-1 / \nu}$. The results in Fig. 10 do not indicate the presence of a regime with $v \approx 0.7$ for $N=200$ and 400 . Instead, for $N=200$, at length scales comparable with $R_{\mathrm{g}}$ we observe ideal linear polymer scaling with $v=1 / 2$, as shown in Fig. 10 by plotting $S(q) q^{2}$. Interestingly, for $N=400$, we observe
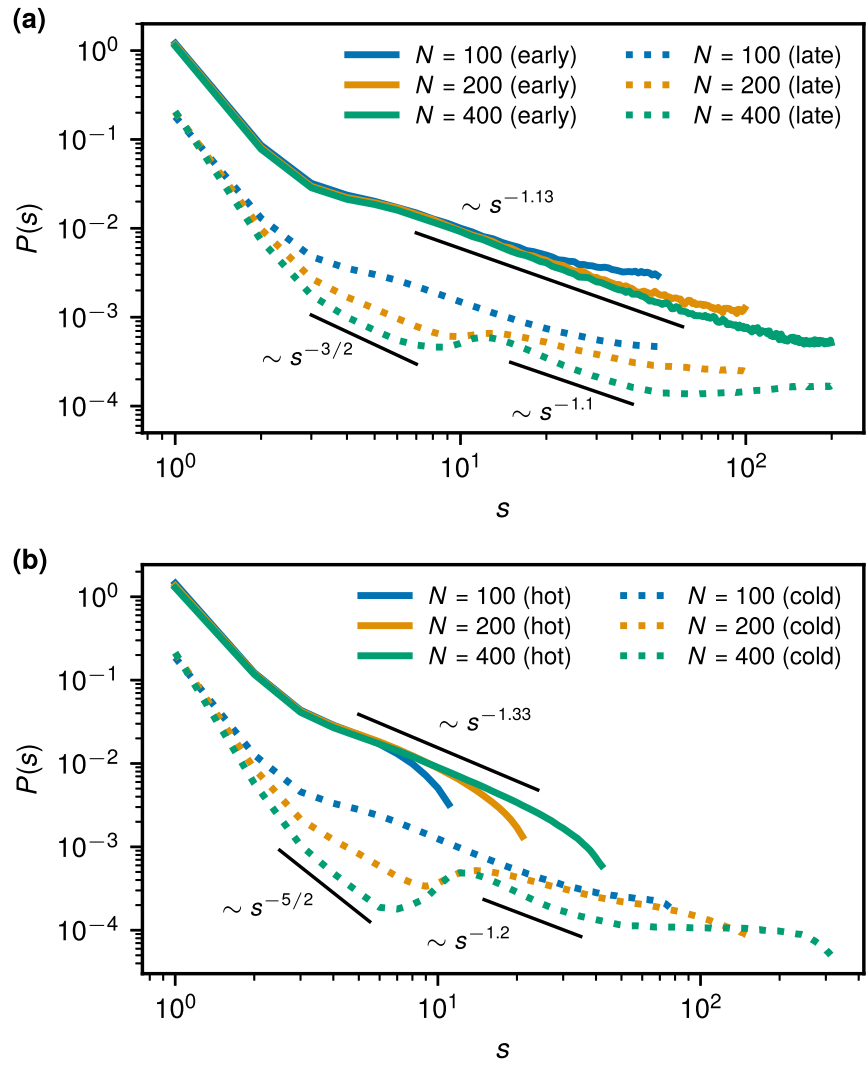

FIG. 11. Contact probability. (a) $P(s)$ of rings is shown for all $N$ at early (solid lines) and late (dotted lines) times. (b) $P(s)$ separately for hot (solid lines) and cold (dotted lines) segments. Here, the $P(s)$ is averaged over the segment's position only within the given segment type. For clarity, dotted curves in (a) and (b) have been shifted vertically. The solid black lines indicated intermediate scaling regimes $P(s) \sim s^{-\gamma}$. Two monomers are considered in contact if they are at a distance smaller than $2^{1 / 6} \sigma$.

scaling $v=0.588$ (see inset of Fig. 10). The self-avoiding walk regime is observed in a relatively narrow range of $q$ and care should be taken as finite-size effects could play a role. However, the superdiffusive dynamics reported in Ref. [24] is consistent with this exponent as well as preliminary results of systems with longer chains (not shown) indicate that this exponent is indeed correct. At lower $q$ that crosses over to $v=$ $1 / 2$ at higher $q$. The behavior of $S(q)$ for $N=100$ is identical to the equilibrium one [3]. Similar results on size scaling with $v=1 / 2$ can be inferred from the mean-square internal distances shown at intermediate contour segment lengths (see Fig. S1 in Ref. [45]).

The ideality of the rings' conformations at smaller length scales can be further inferred from the contact probability $P(s)$, which gives the probability of finding two monomers separated by contour distance $s$ being at a distance smaller than some cutoff value, shown in Fig. 11(a). While for $N=$ 100 , we observe the same behavior as in equilibrium, $N=$ 200 and $N=400$, due to very extended conformations, feature an ideal chain regime with $P(s) \sim s^{-\gamma}$ with $\gamma=3 / 2$ at small $s$, which then crosses over to the equilibriumlike crumpled globule regime with $\gamma$ close to 1 at larger $s$ [3]. The contact probability measured separately for hot and cold 

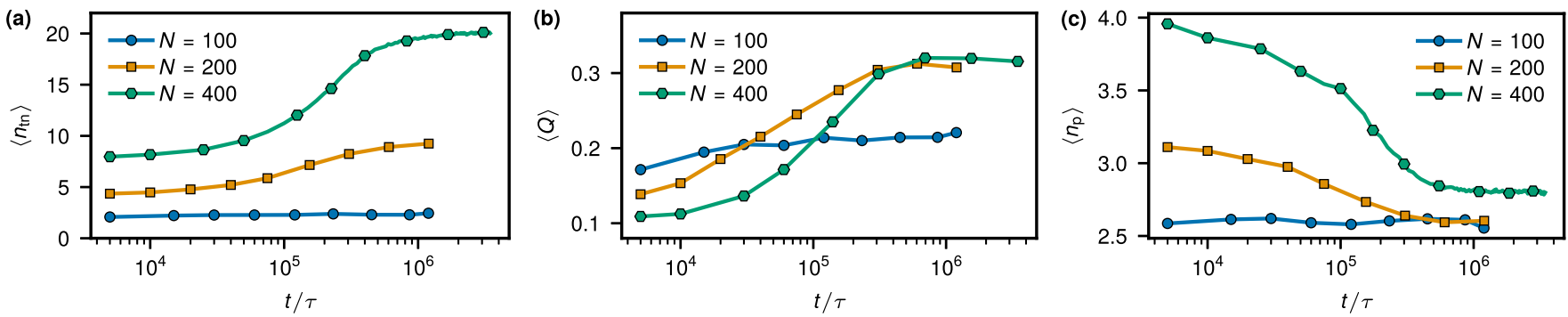

FIG. 12. Time evolution of threading properties. (a), Mean number of threaded neighbors $\left\langle n_{\mathrm{tn}}\right\rangle$ per ring for systems with different $N$ as a function of time after the activity onset. (b), Mean threading length ratio $Q$ as a function of $t$ for different $N$. (c), Mean number of surface penetrations $\left\langle n_{\mathrm{p}}\right\rangle$ as a function of $t$ for different $N$. The system with $N=100$ exhibits equilibrium-like threading at all times.

segments reveals a finer structure (Fig. 11(b)). The exponent $\gamma=4 / 3$ found for hot segment is characteristic for smooth fractal globules, such as collapsed unknotted polymer with smooth outer surface $[15,47]$, which suggests its segregation from the surrounding. This view is confirmed by the finding that $v=1 / 3$ for the hot segment (see Fig. S1(a) in Ref. [45]), because as shown in Ref. [15] $\gamma=2-(d-1) v$ for space-filling fractals in $d$ dimensions. The cold tail shows nonmonotonic behavior with very steep decay at small scales, consistent with very stretched configurations, and shallower decay at longer lengths, that characterize treelike, doubly folded conformations [48]. It also features a peak at $s \approx 10$, which likely stems from the "hairpins" of the double folded segments (see Fig. 1). The latter observation additionally explains why there is a steeper decrease of the contact probability below this length scale and shallower above.

Such dramatic changes in the rings' size and, as a consequence, the number of neighbors, result in a significantly enhanced propensity for inter-ring threading, which we describe now. Inter-ring threadings are quantified using the minimal surface approach. Therein, the ring's contour is considered fixed and is spanned by a surface, whose meancurvature is then minimized, as explained in Refs. [49,50]. Consequently, a threading between a pair of rings is defined by an intersection between one ring's contour and another ring's minimal surface. This approach provides a straightforward geometric picture of the inter-ring threading, is model-independent above the entanglement length [49], and has been employed to quantify the influence of threadings in bulk and confined equilibrium ring polymer melts [20,43,49] or for tadpole-shaped polymers [51]. The depth of threadings is quantified by means of the separation length $L_{\text {sep }}$,

$$
L_{\text {sep }}=\min \left(\sum_{i=\text { even }} L_{\mathrm{t}_{i}}, \sum_{i=\text { odd }} L_{\mathrm{t}_{i}}\right),
$$

where $L_{\mathrm{t}_{i}}$ is the (threading) contour length between the $i$ th and the $(i+1)$ th penetrations of the minimal surface. $L_{\text {sep }}$ quantifies the portion of the threading ring on one side of the threaded ring. Therefore, the ratio $Q=L_{\text {sep }} /\left(N-L_{\text {sep }}\right)$ approximates [51] the relative portion of the threading ring's contour on one side compared to the other side of the threaded ring's surface.

The time evolution of mean threading properties after the activity onset in shown in Fig. 12 for systems with different $N$. Except for $N=100$, the mean number of threaded neighbors $\left\langle n_{\mathrm{tn}}\right\rangle$ per ring, defined as the total number of threadings in the system $n_{\text {tn }}$ divided by $M$, is increasing with time, saturating at a constant value after about $10^{6} \tau$. As expected, $\left\langle n_{\mathrm{tn}}\right\rangle$ correlates strongly with the mean number of ring neighbors within its radius of gyration, $K_{1}\left(R_{\mathrm{g}}\right)$ [compare Fig. 12(a) and Fig. 9(a)]. Although the numbers $K_{1}\left(R_{\mathrm{g}}\right)$ and $\left\langle n_{\mathrm{tn}}\right\rangle$ in the steady state are almost the same, as we checked, the rings contributing to $K_{1}$ are not all threaded and, similarly, some threaded neighbors of a ring are further than $R_{\mathrm{g}}$ from the ring's center of mass. Simultaneously, the threadings are becoming progressively deeper, as evidenced by a rise $\langle Q\rangle$ in Fig. 12(b). Interestingly, even for $N=100$ we observe a slightly increase in $\langle Q\rangle$, albeit no other influence of activity on rings' conformational properties in this system is apparent. While in equilibrium the amount of shallow threading increases with $N$, as quantified by the mean number of penetrations of a ring's minimal surface $\left\langle n_{\mathrm{p}}\right\rangle$, in Fig. 12(c) we show that $\left\langle n_{\mathrm{p}}\right\rangle$ goes down with time for $N=200$ and $N=400$. If only surface penetrations longer than $N_{\mathrm{e}}$ were considered, which provides a modelindependent view on threading statistics [49], $\left\langle n_{\mathrm{p}}\right\rangle$ would actually slightly increase over time [24]. This indicates that many shallow surface piercings disappear, while a few deeper ones appear, which further confirms the increase of threading depth consistent with the evolution of $\langle Q\rangle$. Finally, as we showed in the previous work [24], the mutual ring threadings are responsible for the glassy behavior that emerges from the development of a system-spanning threading cluster.

\section{PHASE SEPARATION}

The gradual change of conformational properties of the rings as well as the slowed dynamics, ensuing from dramatically enhanced threading, coalesce with demixing of cold and hot segments. The hot segments are bonded with the cold ones and, therefore, macrophase separation that would feature a higher resulting temperature contrast [31] is not possible. In fact, we observe the formation of microphase separated regions, with numerous interfaces that mediate the heat flux and decrease the temperature contrast. In the ring systems at hand, block lengths of hot monomers are 13, 25, 50 for $N=100,200,400$, respectively. For the given $\gamma=2 / 3 \tau^{-1}$ and $T_{\mathrm{h}}=3 \epsilon$, the hot block length in the two longer systems would be sufficient to drive phase segregation in an activepassive mixture of linear polymer chains, which irrespective of their type would have length $N_{\mathrm{h}}$. In contrast, such a mixture of chains of length only $N_{h}=13$ (representing an active block 


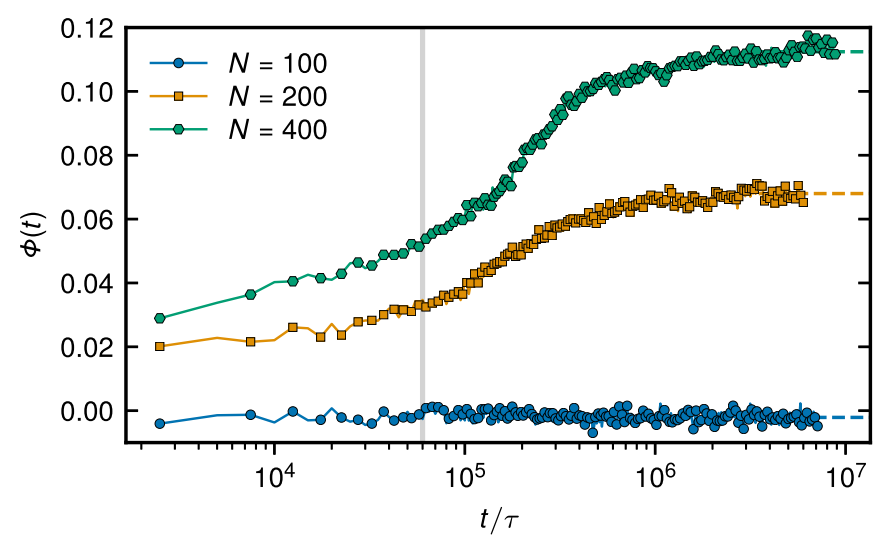

FIG. 13. The phase segregation order parameter. To render comparison across systems with different $N$, the initial value of the order parameter $|\bar{\Phi}(0)|$, corresponding to equilibrium, has been subtracted from $|\bar{\Phi}(t)|$. The gray line indicates the timescale at which significant slowdown of rings' dynamics in the two larger systems becomes apparent. The dashed, colored lines represent the steady-state value of $\Phi$ of a given system.

in the shortest system) would not phase segregate [30,31]. Nevertheless, it is an open question whether demixing occurs in systems with polymers of unequal length $N_{\mathrm{h}}$ and $N_{\mathrm{c}}$ at a similar temperature contrast. To quantify phase separation in our systems, we compute the order parameter $\Phi(t)=$ $x(t) / x(0)-1$, where $x(t)$ is the particle-averaged number fraction of interchainlike particles in a $r_{\mathrm{c}}=2^{1 / 6} \sigma$ neighborhood of a given particle at a given time $t$, and $x(0)$ is the same quantity at $t=0 \tau$ corresponding to equilibrium (at $t=0 \tau$ distinct types were assigned to particles of an equilibrium melt state, but equations of motions were not integrated yet; thus, such state must be reasonably mixed). Such choice of $\Phi(t)$ allows us to explicitly compare systems with different fractions of hot monomers along the chain, which are characterized by different values of $x(0)$.

As reported in Fig. 13, while the system with $N=100$, which is equilibriumlike and does not vitrify, does not show strong propensity for segregation of cold and hot monomers, the two larger systems do. For $N=200$ and $N=400$, immediately after the activity onset, the cold and hot segments in these systems become somewhat segregated $\left(t<10^{5} \tau\right)$. This agrees well with reduced density around the hot segments and their enhanced diffusion, as reported in Fig. 4. As the rings become gradually more and more constrained by numerously increased threadings, further separation continues up to around few millions $\tau$. The system with $N=400$ arrives at a more segregated state than the one with $N=200$.

The effective temperatures responsible for the phase segregation are obtained as an average over particles of the same species. To a good approximation, the velocity distribution of hot (cold) particles can be described using the MaxwellBoltzmann distribution with temperature $T_{\mathrm{h}(\mathrm{c})}^{\mathrm{eff}}$ [31]. In a phase-segregated state, the deviations from the corresponding Maxwell-Boltzmann distribution arise from particles that sit at the interfaces and, on average, have an intermediate effective temperature, $T_{\mathrm{c}}^{\mathrm{eff}}<T^{\mathrm{eff}}<T_{\mathrm{h}}^{\text {eff }}$. In the steady state, the total average power supplied to the system from the thermostats through random collisions $\dot{E}^{\text {rand }}=-3 \gamma\left(n_{\mathrm{h}} T_{\mathrm{h}}+n_{\mathrm{c}} T_{\mathrm{c}}\right)$ should balance the total dissipated energy per unit time through friction $\dot{E}^{\text {fric }}=3 \gamma\left(n_{\mathrm{h}} T_{\mathrm{h}}^{\text {eff }}+n_{\mathrm{c}} T_{\mathrm{c}}^{\text {eff }}\right)$ [30]. Therefore,

$$
n_{\mathrm{h}} T_{\mathrm{h}}^{\mathrm{eff}}+n_{\mathrm{c}} T_{\mathrm{c}}^{\mathrm{eff}}=n_{\mathrm{h}} T_{\mathrm{h}}+n_{\mathrm{c}} T_{\mathrm{c}} .
$$

The relation Eq. (13) is satisfied for all $N$ at any point in time $t$, although the two larger systems feature substantial conformational rearrangements at early times. The reason for this is the fact that Eq. (13) works as long as no external work is being produced, which is the case in the systems considered. Note that dividing Eq. (13) by $N$ provides the equality between the mean effective temperature, governing the center of mass dynamics of the ring, and the mean thermostat temperature. This makes it possible to apply the single chain results like in section A to the melt case where the effect of the other chains, besides the topological constraints, is just in adjusted value of the effective temperatures.

As shown in Fig. 14(a), phase separation in the systems with $N=200$ and $N=400$ leads to a gradually increased discrepancy between the two effective temperatures in the system. Nevertheless, the ratio $T_{\mathrm{h}}^{\mathrm{eff}} / T_{\mathrm{c}}^{\mathrm{eff}} \approx 1.6-1.8$ in these systems is still much smaller than the one imposed by the thermostat $T_{\mathrm{h}} / T_{\mathrm{c}}=3$. The propensity of hot blocks to phase segregate in these systems locally reduces density around them and therefore disproportionately increases their diffusivity, as compared to the cold segments. This results in an oriented reptationlike motion, where partially active rings are essentially driven by hot segment displacements, which promotes the formation of interring threading constraints and makes unthreading events less likely. As the rings get more constrained by their threading neighbors (Fig. 12), they continue to locally phase segregate stronger (Fig. 13), which again increases the difference between segment diffusivities and therefore makes the unthreading processes even more unlikely. Thus, a formed glassy state is maintained by the nonequilibrium microphase separation. In contrast, the smallest system with $N=100$ that is not segregating stronger features more contacts between hot and cold monomers and, therefore, heats up the cold subsystem at the expense of the hot one, resulting in $T_{\mathrm{h}}^{\text {eff }} / T_{\mathrm{c}}^{\text {eff }} \approx 1.4$. The rings as a whole in this system follow an equilibriumlike dynamics with diffusivities of hot and cold segments being proportional to an overall enhanced temperature, as detailed in Sec. III. Lastly, for all $N$, the fact that cold and hot segments are bonded creates intermediate regions with temperature smaller than $T_{\mathrm{h}}^{\text {eff }}$ but larger than $T_{\mathrm{c}}^{\mathrm{eff}}$. This effect is quantified in Fig. 14(c), where we show the distribution of effective temperatures along the ring's contour $T^{\text {eff }}(s)$.

The discrepancy between the incoming and outgoing heat fluxes in the cold and hot subsystems results in a nonvanishing entropy production $\dot{S}$ per particle,

$$
\dot{S}=\frac{3 \gamma}{N} \sum_{s=0}^{N}\left\langle\frac{T^{\mathrm{eff}}(s)}{T(s)}-1\right\rangle,
$$

where $\langle\ldots\rangle$ is the ensemble average over different polymer chains in the system. In Eq. (14), we explicitly take into account deviations from $T_{\mathrm{h}(\mathrm{c})}^{\mathrm{eff}}$ for boundary monomers. The time evolution of $\dot{S}$ for different $N$ is shown in the inset of Fig. 14(c). For $N=100, \dot{S}$ is the highest, indicating the least 

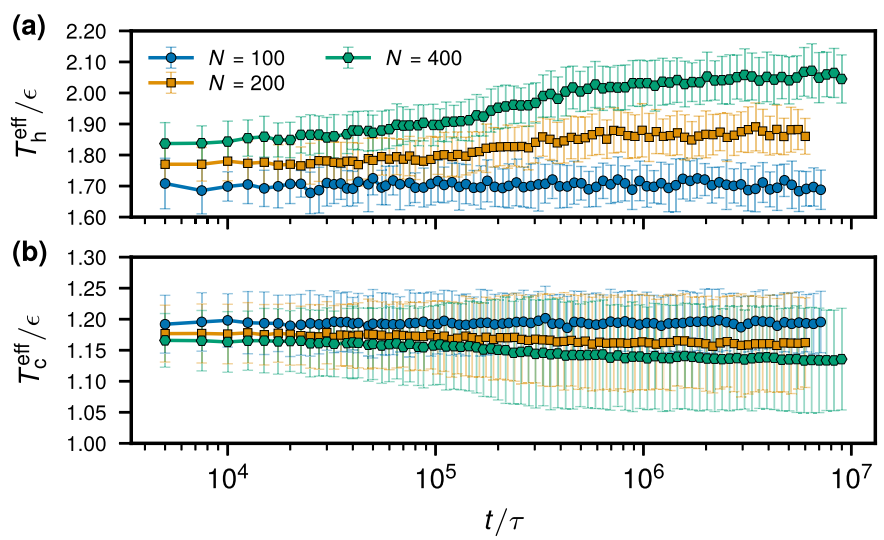

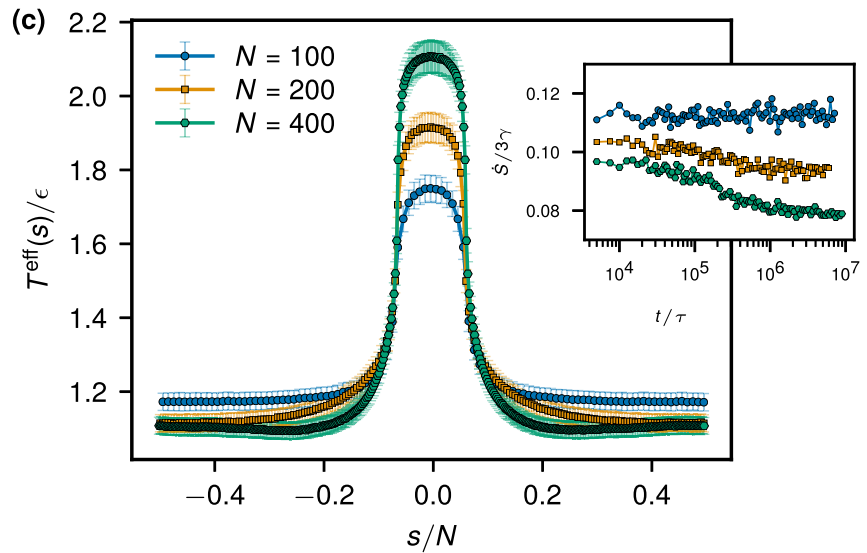

FIG. 14. Effective temperatures. Time evolution of the system-averaged effective temperature of hot, $T_{\mathrm{h}}^{\text {eff }}$, (a) and cold, $T_{\mathrm{c}}^{\text {eff }}$, (b) monomers. (c) Distribution of the effective temperature along the ring's contour in the steady state for different $N$. ( $s$ is centered at the middle of the active segment). Inset: Time evolution of the mean entropy production $\dot{S}$ per monomer Eq. (14) (error bars are not shown for clarity; generally, the standard deviation of $\dot{S} / 3 \gamma$ is around 0.15 is all cases). In (a)-(c), error bars indicate the standard deviation of the corresponding quantity.

degree of phase separation. For $N=200$ and $N=400, \dot{S}$ decreases with time, highlighting further phase separation of hot and cold monomers in these systems. The fact that $\dot{S}$ is the smallest for $N=400$ further confirms that it is the most phase separated. In addition, by neglecting the contour distribution of temperature, Eq. (14) can be recast as

$$
\dot{S} / 3 \gamma \simeq n_{\mathrm{h}}\left(\frac{T_{\mathrm{h}}^{\text {eff }}}{T_{\mathrm{h}}}-1\right)+n_{\mathrm{c}}\left(\frac{T_{\mathrm{c}}^{\mathrm{eff}}}{T_{\mathrm{c}}}-1\right),
$$

where $n_{\mathrm{c}}$ and $n_{\mathrm{h}}$ are the fractions of cold and hot monomers, respectively. Now, using the heat balance Eq. (13), Eq. (15) transforms into

$$
\dot{S} / 3 \gamma \simeq n_{\mathrm{c}}\left(\frac{T_{\mathrm{h}}}{T_{\mathrm{c}}}-1\right)\left(\frac{T_{\mathrm{c}}^{\mathrm{eff}}}{T_{\mathrm{c}}}-1\right) .
$$

Stronger tendency toward phase segregation reduces the number of contacts between cold and hot monomers, thereby making the effective temperature of cold particles [Fig. 14(b)] and, correspondingly, $\dot{S}$ lower, as already observed at very short simulation times. Equation (16) further confirms that $\dot{S}(N=100)>\dot{S}(N=200)>\dot{S}(N=400)$, as observed directly in Fig. 14(c). Finally, the microphase separated systems are composed of a less dense active phase and more dense passive phase. This is illustrated in Fig. 15, showing anticorrelation between temperature and density distributions within the simulation box for $N=100$ and $N=400$.

A similar system of linear chains becomes less phase separated than the one of rings. We show this by taking a steady-state ring configuration $\left(t=2 \times 10^{6} \tau\right)$, cutting all chains in the middle of the cold segment, which results in ABA triblocks, and evolving such a system further on, as shown in Fig. 16. We also observe an increase in the entropy production with a lesser difference between the observed effective cold and hot temperatures, in line with reduced degree of phase separation. Interestingly, the chain ends have more frequent contacts with hot regions that results in their higher effective temperature, as seen in Fig. 16(b). Finally, in the two larger systems of cut rings that have a propensity for demixing, the oriented reptationlike motion driven by the hot segment persists. An interesting question that remains is whether such ABA (cold-hot-cold) triblocks can form ordered structures that correspond to the strongly phase-segregated limit in equilibrium block copolymers [52]. For active copolymer rings, which we have now extensively discussed, such behavior is obviously suppressed due to the topological glass formation, which disables the rings to potentially segregate more due to the formed topological constraints. From the evolution of the phase-separation order parameter for ABA triblocks [see Fig. 16(a)] and from the final system states [see inset of Fig. 16(b)], we do not observe the formation of ordered structures. The phase-separated regions of active monomers rather reminisce of the weakly segregated regime for equilibrium copolymers. It is instructive to compare the present nonequilibrium ABA systems with the equilibrium ones [53]. In particular, for the three ABA systems considered, we find the nonequilibrium incompatibility parameter $\chi=$ $T_{\mathrm{h}}^{\text {eff }} / T_{\mathrm{c}}^{\text {eff }}-1$ to be $0.42,0.51$, and 0.68 for $N=100,200$, and 400 , respectively. Equilibrium diblocks with a similar fraction of A monomers $\left(f_{\mathrm{A}}=7 / 8\right)$ would form a spherical (micellar) phase at comparable values of (equilibrium) $\chi$-parameters for the two bigger $N$ [53]. In our case, however, we do not observe a tendency for such behavior. Note, however, that this comparison is for illustration only, as it is not guaranteed that the phase diagrams are similar and it is not clear now if active copolymers can form ordered structures at all.

A mixture of $M$ cold chains of length $N_{\mathrm{c}}$ and $M$ hot chains of length $N_{\mathrm{h}}$ shows a stronger propensity toward phase segregation, as seen in Fig. 17(a). As recently derived by Ilker and Joanny [35], the critical ratio $\chi^{*}$ needed for segregation $\left(\chi=T_{\mathrm{h}}^{\text {eff }} / T_{\mathrm{c}}^{\text {eff }}-1\right)$ scales as

$$
\chi^{*}=\alpha\left(N_{\mathrm{h}}^{-1 / 2}+N_{\mathrm{c}}^{-1 / 2}\right),
$$

with chain lengths (note that the right-hand side is not squared, as it would be for equilibrium phase separation). This agrees well with earlier simulation results on phase separation in symmetric active-passive mixtures [30] [Fig. 17(b)]. Furthermore, we compared the $\chi$ parameter in the three present cases with the critical line Eq. (17), with $\alpha$ extracted from Ref. [30]. 

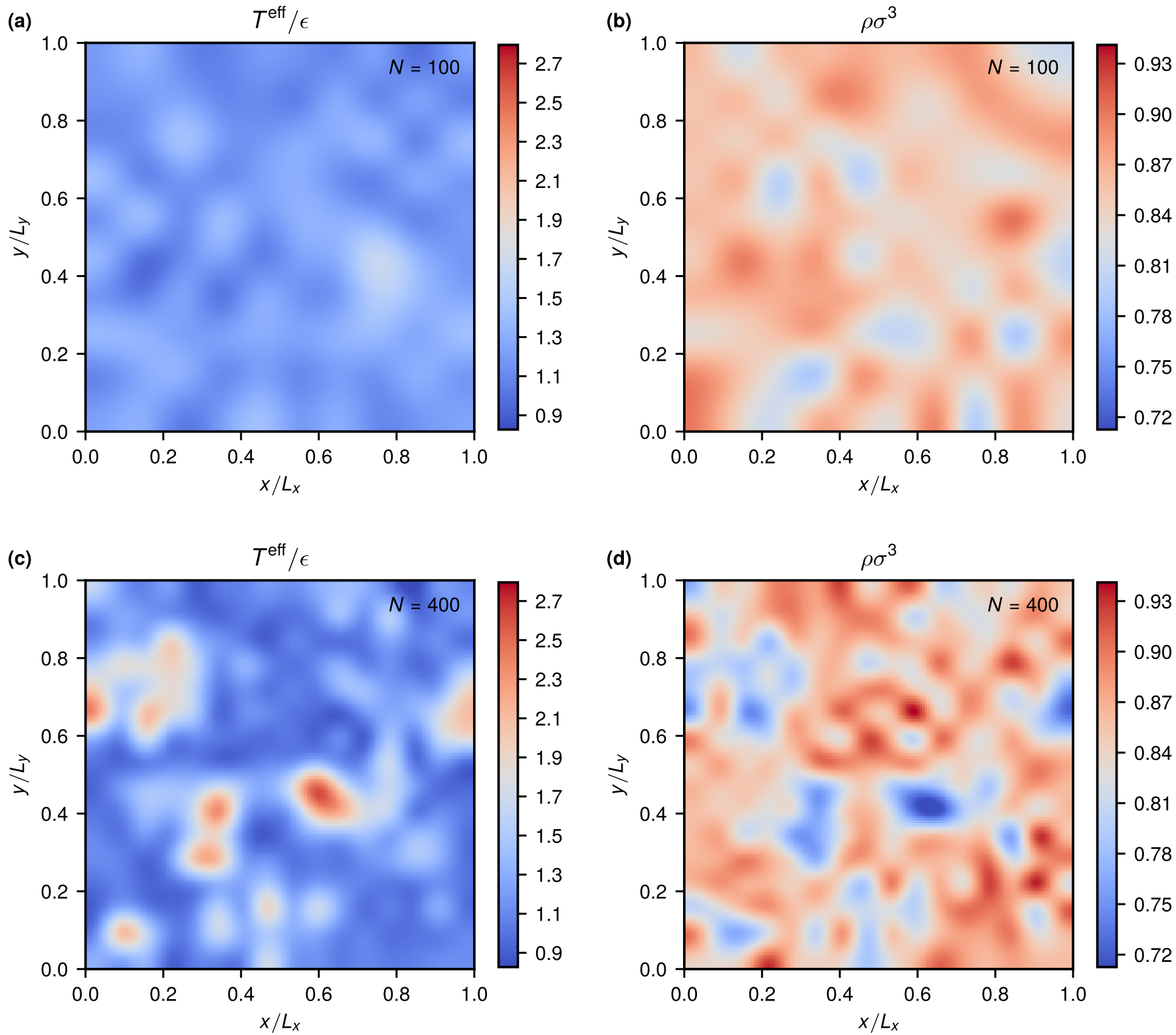

FIG. 15. Spatial distribution of temperature and density. $T^{\text {eff }}$ and $\rho$ are shown for $N=100[$ [a), (b)] and $N=400$ [(c), (d)]. To compute the distributions, the systems were divided into $n_{\mathrm{b}}^{3}$ small sub-boxes of comparable size $\sigma_{\mathrm{b}} \approx 5.7 \sigma$. One layer corresponding to a fixed $z$ elevation is shown. The heat maps for $N=100$ are normalized with the same scale as for $N=400$.

We find that the system with $N_{\mathrm{h}}=13$ and $N_{\mathrm{c}}=87$, unlike the other two, lies below the critical line [Fig. 17(b)]. This further supports that the two systems of active-passive mixtures of linear chains of lengths equivalent to block lengths in our ring systems do phase separate while the shortest one does not, highlighting the role of the nonequilibrium phase separation in the process of formation of the active topological glass.

\section{SUMMARY AND CONCLUDING REMARKS}

In summary, we have considered in detail melts of unknotted and nonconcatenated block copolymer rings driven out of equilibrium by different thermostats applied to the two different blocks. We focused on systems with different ring length $N$, while keeping the same length ratio of active blocks $N_{\mathrm{h}}=N / 8$ coupled to a heat bath at the temperature $T_{\mathrm{h}}=3 T_{\mathrm{c}}$.
We have shown that at early times the segmental and center of mass dynamics is equilibriumlike with an effectively higher temperature. In particular, in the shortest system with $N=$ 100 , the presence of activity effectively increases the ring's diffusivity, as given by Eq. (A3), however, its conformational and threading properties remain essentially unchanged. On the contrary, in the case of $N=200$ and $N=400$, already shortly after the activity onset, the hot segments diffuse faster as compared to the relation Eq. (A3). This arises due to a somewhat lower density around them that hallmarks an early stage of nonequilibrium phase separation. The discrepancy in segment dynamics in this case results in an oriented reptationlike regime, where rings are essentially pulled by the hot-segment displacements, which promotes the formation of topological constraints until the motion of rings stops, forming a very slowly relaxing glassy state that is built on topological 

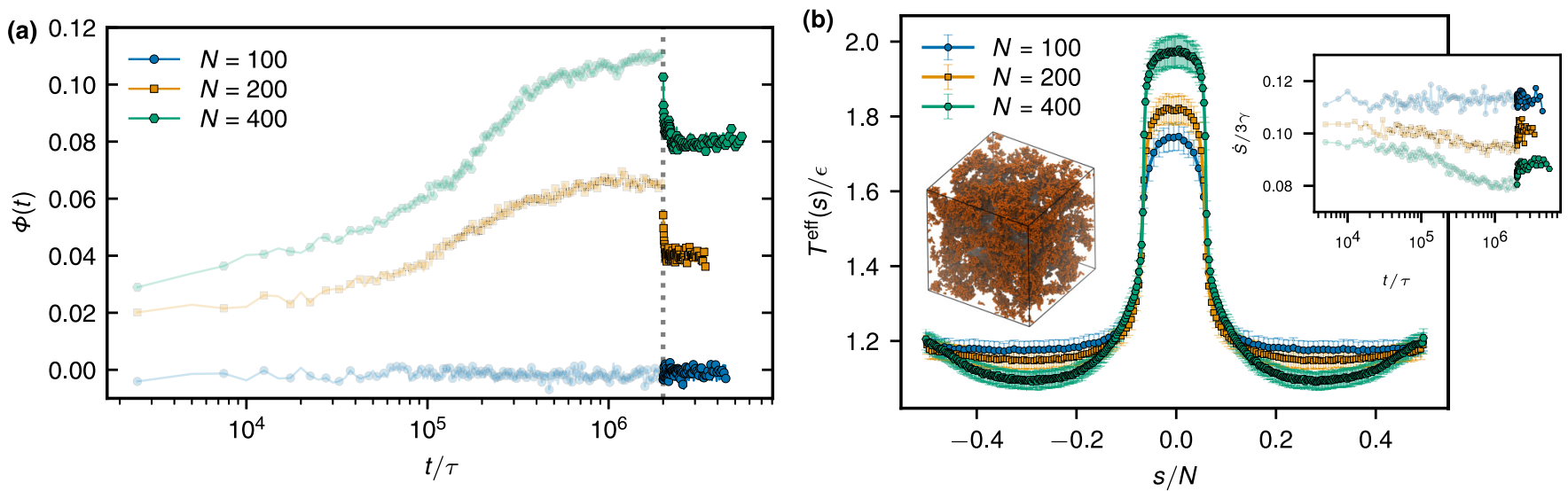

FIG. 16. Nonequilibrium phase segregation of cut rings (ABA triblocks). (a) Time evolution of the phase-segregation order parameter for rings (transparent curves) before $t<2 \times 10^{6} \tau$ and linear polymer chains (opaque curves) obtained after cutting the rings in the middle of the cold segment at $t=2 \times 10^{6} \tau$ (dotted gray line) for different $N$. (b) Distribution of the effective temperature along the contour of cut rings in the steady state (after equilibrating for $1.5 \times 10^{6} \tau$ ) for different $N$ ( $s$ is centered at the middle of the active segment). Inset: Time evolution of the mean entropy production $\dot{S}$ for rings before (transparent) and after (opaque) cutting. The snapshot on the left illustrates the partially segregated state of hot monomers (cold monomers are not shown for clarity) in the system with $N=400$ at the end of the simulation. In (b), error bars indicate the standard deviation of $T^{\mathrm{eff}}(s)$.

constraints and activity. The critical diffusivity contrast for the glass formation seems to coincide with the phase separation contrast, but a more detailed study is necessary to determine the relation exactly.

The present nonequilibrium rings, in a sense, represent a hybrid between ring and linear polymers. On the one hand, they are strongly influenced by threading constraints, lack ends, and, therefore cannot reptate as linear chains. However, for strong enough temperature discrepancies, the active segments effectively serve as a chain's end, exploring neighboring sites and dragging the rest of the polymer with itself. Such dynamics gives rise to elongated, doubly folded conformations that locally feature statistics of linear polymers.

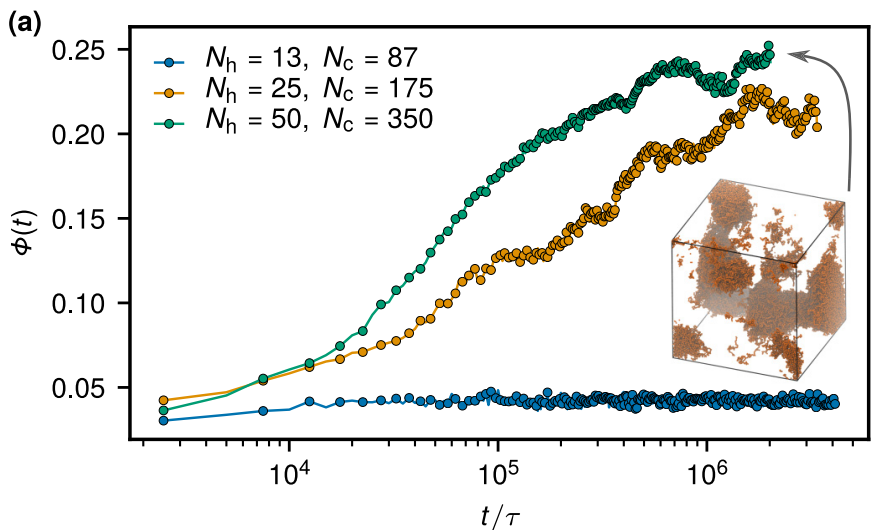

Many intriguing questions remain to be answered for the active topological glass. Some of these pertain to other activity models. For instance, in a dilute mixture of self-propelling particles [29] with different propulsion speeds $v$, densities $\rho$ adjust to equalize the momentum density $\rho v$, not the pressure $p \sim \rho v^{2}$, across the system. Before the separation, this results in a less dense phase of particles with higher mobility and, therefore, higher pressure, and a more dense phase of lower mobility particles with lower pressure. Similarly, in a segregated two-temperature mixture $[30,31,34,35]$, the phase with particles in contact with a hotter thermostat, locally exerting higher pressure, is more dilute as compared to the phase with colder particles. As the microscopic and phase separation dynamics is different for the thermostat-driven particle

FIG. 17. Nonequilibrium phase segregation of linear polymer chains of different length. (a) Time evolution of the phase-segregation order parameter for linear polymers mixtures with $M=1600$ hot chains of length $N_{\mathrm{h}}=N / 8$ and $M=1600$ cold chains of length $N_{\mathrm{c}}=N-N_{\mathrm{h}}$ for $N=100,200$, and 400. The inset snapshot shows only the hot part of the system with $N=400$ at late times. (b) The nonequilibrium incompatibility parameter $\chi=T_{\mathrm{h}}^{\text {eff }} / T_{\mathrm{c}}^{\text {eff }}-1$ as a function of $N_{\mathrm{c}}^{-1 / 2}+N_{\mathrm{h}}^{-1 / 2}$ for the three systems considered (circles). The gray dashed line is the critical line, $\chi^{*}=\alpha\left(N_{\mathrm{c}}^{-1 / 2}+N_{\mathrm{h}}^{-1 / 2}\right)$, with $\alpha=1.746 \pm 0.081$, extracted from symmetric linear polymer mixtures in Ref. [30]. The five black squares are the measured values of $\chi^{*}$ from Ref. [30] for symmetric $\left(N_{\mathrm{h}}=N_{\mathrm{c}}=10,20,40,70\right.$ and 100) mixtures of equal number of hot and cold linear chains. The theory [35] supports the numerical results. 
systems in comparison to systems with self-propelled particles, it would be interesting to examine the existence of the active topological glass for the latter model. To our knowledge, the nonequilibrium phase separation of polymers composed out of self-propelling particles has not been investigated systematically and certainly it would be interesting to test whether the $N^{-1 / 2}$ scaling of the incompatibility parameter holds in such systems as well.

Other properties of the active topological glass are yet to be elucidated. In particular, the exact kinetics of the formation of the active topological glass, its response to external shear stress, as well as the strength of the activity quench on the rings' segments necessary to observe the arrested states are unknown. A proper description of this phenomenon requires an understanding of the nonequilibrium microphase separation. It is very interesting, for example, whether nonequilibrium active diblock polymers can form ordered structures, such as lamellae, cylinders, or micelles, as is typical for segregating diblock copolymers in equilibrium [52,54]. The equilibrium melt of a diblock or, in the case of the cut rings a simple triblock, copolymers of these lengths would form micelles. In our partly active systems, we do not observe these structures, however, this might not only be because the glassy state arrests the phase separation, but also the incompatibility parameter and the chain dynamics are different from equilibrium and, therefore, the phase diagram is yet to be explored. A more complete understanding of these phenomena might further trigger development of active materials with novel properties and shed light on self-organization and dynamics of different biological polymers, in particular chromatin, that are subject to heterogeneous activity along their contours and can feature a loopy structure at different length scales. In this context, the main question pertains on the physical mechanism that governs the separation of transcriptionally active (euchromatin) and the passive (heterochromatin) fibers. Since both species also exhibit chemical differences, it is difficult to establish if the equilibrium microphase separation or the nonequilibrium analog plays the pivotal role. Moreover, the potential topoisomerase-induced crossability and the topology of the chromatin fiber affects the accessible morphologies. In this paper, we pinpointed the differences in the steady-state morphologies (suppressed formation of strongly separated structures) and the segment dynamics (oriented reptation) that can guide the experiments to discern these mechanisms.

\section{ACKNOWLEDGMENTS}

J.S. and I.C. acknowledge support from the Austrian Science Fund (FWF) through the Lise-Meitner Fellowship No. M 2470-N28. I.C. acknowledges the Mobility Fellowship provided by the Vienna Doctoral School in Physics (VDSP). The authors would like to acknowledge networking support by the COST Action CA17139. We are grateful for generous computational time at Vienna Scientific Cluster and Max Planck Computing and Data Facility. This work has been supported by the European Research Council under the European Union's Seventh Framework Programme (FP7/20072013)/ERC Grant Agreement No. 340906-MOLPROCOMP. This research was supported in part by the National Science
Foundation under Grant No. NSF PHY-1748958 and NIH Grant No. R25GM067110. J.S. and K.K. acknowledge the program Biological Physics of Chromosomes KITP UCSB, 2020 , for providing a discussion forum this work benefited from.

\section{APPENDIX A: CENTER OF MASS DIFFUSION OF A SINGLE CHAIN COUPLED TO TWO THERMOSTATS}

Consider a single polymer chain of $N$ monomers out of which $N_{\mathrm{h}}$ are connected to a Langevin thermostat at $T_{\mathrm{h}}$ and $N_{\mathrm{c}}$ monomers are connected to a Langevin thermostat at $T_{\mathrm{c}}$. The Langevin equation for the $i$ th monomer is

$$
m \dot{\mathbf{v}}_{i}=-m \gamma \mathbf{v}_{i}-\nabla_{i} U+\left(2 m \gamma T_{i}\right)^{1 / 2} \eta_{i},
$$

where $\gamma$ is the thermostat coupling parameter, $m$ is the monomer's mass, $U$ is the interparticle interaction potential, each component of $\boldsymbol{\eta}_{i}$ is a Gaussian random variable satisfying $\left\langle\eta_{i, \alpha}(t) \eta_{j, \beta}\left(t^{\prime}\right)\right\rangle=\delta_{i j} \delta_{\alpha \beta} \delta\left(t-t^{\prime}\right)$. The temperature $T_{i}$ is $T_{\mathrm{c}}$ or $T_{\mathrm{h}}$ depending on the bead $\left(k_{\mathrm{B}}=1\right)$. By summing Eq. (A1) over all monomers, we get the equation for the center of mass velocity, $\mathbf{v}$ :

$$
M \dot{\mathbf{v}}=-M \gamma \mathbf{v}+\left(2 M \gamma T_{\text {eff }}\right)^{1 / 2} \eta,
$$

where $M=m N$ and $T_{\text {eff }}=\left(T_{\mathrm{c}} N_{\mathrm{c}}+T_{\mathrm{h}} N_{\mathrm{h}}\right) / N$. Note that the friction coefficient $M \gamma \equiv m \gamma N$ in Eq. (A2) corresponds to the Rouse model of polymer dynamics [37]. The result Eq. (A2) follows from the fact that $\mathbf{v}=N^{-1} \sum_{i}^{N} \mathbf{v}_{i}, \sum_{i}^{N} \nabla_{i} U=0$, and that the sum of independent Gaussian random variables with unit variance and zero mean is again a Gaussian random variable with zero mean but a larger variance, namely, $\sum_{i}^{k} \eta_{i}=$ $k^{1 / 2} \eta$. This means that the center of mass of the chain moves as a Langevin particle with the effective temperature $T_{\text {eff }}$ and effective diffusion coefficient

$$
D_{\text {eff }}=\frac{T_{\text {eff }}}{M \gamma}=\frac{T_{\mathrm{c}} N_{\mathrm{c}}+T_{\mathrm{h}} N_{\mathrm{h}}}{m \gamma N^{2}}=\frac{D_{\mathrm{c}} N_{\mathrm{c}}+D_{\mathrm{h}} N_{\mathrm{h}}}{N^{2}},
$$

where $D_{\mathrm{c}}$ and $D_{\mathrm{h}}$ are diffusion coefficients of hot and cold monomers, respectively. Note that the result is independent of the ordering of the monomers.

TABLE II. Size and shape properties of equilibrium rings and linear chains. $\left\langle R_{\mathrm{g}}^{2}\right\rangle$ is the mean-square radius of gyration, $\left\langle R_{\mathrm{e}}^{2}\right\rangle$ in the case of rings is the mean-square distance between two monomers separated by the contour length $N / 2$, while in the case of linear polymers it is the mean-square end-to-end distance and $\lambda_{i}, i=1,2,3$ are the eigenvalues of the gyration tensor ordered such that $\lambda_{1} \geqslant$ $\lambda_{2} \geqslant \lambda_{3}$. The value in the parentheses indicates the standard error. The data is adapted from Ref. [3].

\begin{tabular}{lccccc}
\hline \hline Topology & $N$ & $\left\langle R_{\mathrm{g}}^{2}\right\rangle / \sigma^{2}$ & \multicolumn{1}{c}{$\left\langle R_{\mathrm{e}}^{2}\right\rangle / \sigma^{2}$} & $\left\langle\lambda_{1}\right\rangle /\left\langle\lambda_{3}\right\rangle$ & $\left\langle\lambda_{2}\right\rangle /\left\langle\lambda_{3}\right\rangle$ \\
\hline Ring & 100 & $17.2(0.4)$ & $50.8(1.5)$ & 6.4 & 2.3 \\
& 200 & $30.8(0.7)$ & $88.8(2.7)$ & 5.9 & 2.2 \\
& 400 & $52.9(1.2)$ & $149.4(4.8)$ & 5.5 & 2.1 \\
Linear & 100 & $43.4(1.2)$ & $263.8(1.6)$ & 12.9 & 2.8 \\
& 200 & $88.9(1.2)$ & $538.9(1.6)$ & 12.6 & 2.8 \\
& 400 & $180.8(1.3)$ & $1095.3(1.6)$ & 12.3 & 2.8 \\
\hline \hline
\end{tabular}




\section{APPENDIX B: SHAPE PROPERTIES OF EQUILIBRIUM RINGS AND LINEAR CHAINS}

In this Appendix, we summarize in Table II the size and shape parameters of equilibrium ring and linear polymer chains in the melt that are mentioned in Sec. IV of the main paper.

[1] N. T. Moore, R. C. Lua, and A. Y. Grosberg, Topologically driven swelling of a polymer loop, Proc. Natl. Acad. Sci. USA 101, 13431 (2004).

[2] M. Kapnistos, M. Lang, D. Vlassopoulos, W. PyckhoutHintzen, D. Richter, D. Cho, T. Chang, and M. Rubinstein, Unexpected power-law stress relaxation of entangled ring polymers, Nat. Mater. 7, 997 (2008).

[3] J. D. Halverson, W. B. Lee, G. S. Grest, A. Y. Grosberg, and K. Kremer, Molecular dynamics simulation study of nonconcatenated ring polymers in a melt. I. Statics, J. Chem. Phys. 134, 204904 (2011).

[4] J. D. Halverson, W. B. Lee, G. S. Grest, A. Y. Grosberg, and K. Kremer, Molecular dynamics simulation study of nonconcatenated ring polymers in a melt. II. Dynamics, J. Chem. Phys. 134, 204905 (2011).

[5] A. R. Brás, S. Gooßen, M. Krutyeva, A. Radulescu, B. Farago, J. Allgaier, W. Pyckhout-Hintzen, A. Wischnewski, and D. Richter, Compact structure and non-gaussian dynamics of ring polymer melts, Soft Matter 10, 3649 (2014).

[6] S. Gooßen, A. R. Brás, M. Krutyeva, M. Sharp, P. Falus, A. Feoktystov, U. Gasser, A. Wischnewski, and D. Richter, Molecular Scale Dynamics of Large Ring Polymers, Phys. Rev. Lett. 113, 168302 (2014).

[7] D. Vlassopoulos, Macromolecular topology and rheology: beyond the tube model, Rheol. Acta 55, 613 (2016).

[8] T. Iwamoto, Y. Doi, K. Kinoshita, Y. Ohta, A. Takano, Y. Takahashi, M. Nagao, and Y. Matsushita, Conformations of ring polystyrenes in bulk studied by SANS, Macromolecules 51, 1539 (2018).

[9] F. Ferrari and I. Lazzizzera, Polymer topology and ChernSimons field theory, Nucl. Phys. B 559, 673 (1999).

[10] A. Narros, A. J. Moreno, and C. N. Likos, Influence of topology on effective potentials: Coarse-graining ring polymers, Soft Matter 6, 2435 (2010).

[11] A. Narros, A. J. Moreno, and C. N. Likos, Effective interactions of knotted ring polymers, Biochem. Soc. Trans. 41, 630 (2013).

[12] A. Narros, C. N. Likos, A. J. Moreno, and B. Capone, Multiblob coarse graining for ring polymer solutions, Soft Matter 10, 9601 (2014).

[13] A. Narros, A. J. Moreno, and C. N. Likos, Effects of knots on ring polymers in solvents of varying quality, Macromolecules 46, 3654 (2013).

[14] I. Chubak, E. Locatelli, and C. N. Likos, Ring polymers are much stronger depleting agents than linear ones, Mol. Phys. 116, 2911 (2018).

[15] J. D. Halverson, J. Smrek, K. Kremer, and A. Y. Grosberg, From a melt of rings to chromosome territories: the role of topological constraints in genome folding, Rep. Prog. Phys. 77, 022601 (2014).
[16] A. R. Klotz, B. W. Soh, and P. S. Doyle, Equilibrium structure and deformation response of $2 \mathrm{D}$ kinetoplast sheets, Proc. Natl. Acad. Sci. USA 117, 121 (2020).

[17] K. S. Chichak, S. J. Cantrill, A. R. Pease, S.-H. Chiu, G. W. V. Cave, J. L. Atwood, and J. F. Stoddart, Molecular borromean rings, Science 304, 1308 (2004).

[18] S. P. Obukhov, Topologically Induced Glass Transition in Freely Rotating Rods, are slides from a talk at Kavli Institute for Theoretical Physics Santa Barbara, Accessed April 2019 (1997), http://online.kitp.ucsb.edu/online/jamming2/obukhov/.

[19] E. Lee, S. Kim, and Y. Jung, Slowing down of ring polymer diffusion caused by inter-ring threading, Macromol. Rapid Commun. 36, 1115 (2015).

[20] J. Smrek and A. Y. Grosberg, Minimal surfaces on unconcatenated polymer rings in melt, ACS Macro Lett. 5, 750 (2016).

[21] D. G. Tsalikis, V. G. Mavrantzas, and D. Vlassopoulos, Analysis of slow modes in ring polymers: Threading of rings controls long-time relaxation, ACS Macro Lett. 5, 755 (2016).

[22] D. Michieletto and M. S. Turner, A topologically driven glass in ring polymers, Proc. Natl. Acad. Sci. U.S.A 113, 5195 (2016).

[23] D. Michieletto, N. Nahali, and A. Rosa, Glassiness and heterogeneous dynamics in dense solutions of ring polymers, Phys. Rev. Lett. 119, 197801 (2017).

[24] J. Smrek, I. Chubak, C. N. Likos, and K. Kremer, Active topological glass, Nat. Commun. 11, 26 (2020).

[25] A. Zidovska, D. A. Weitz, and T. J. Mitchison, Micron-scale coherence in interphase chromatin dynamics, Proc. Natl. Acad. Sci. USA 110, 15555 (2013).

[26] R. Bruinsma, A. Grosberg, Y. Rabin, and A. Zidovska, Chromatin hydrodynamics, Biophys. J. 106, 1871 (2014).

[27] S. Wu, K. M. Turner, N. Nguyen, R. Raviram, M. Erb, J. Santini, J. Luebeck, U. Rajkumar, Y. Diao, B. Li et al., Circular ecDNA promotes accessible chromatin and high oncogene expression, Nature 575, 699 (2019).

[28] A. P. Solon, J. Stenhammar, R. Wittkowski, M. Kardar, Y. Kafri, M. E. Cates, and J. Tailleur, Pressure and Phase Equilibria in Interacting Active Brownian Spheres, Phys. Rev. Lett. 114, 198301 (2015).

[29] A. P. Solon, Y. Fily, A. Baskaran, M. E. Cates, Y. Kafri, M. Kardar, and J. Tailleur, Pressure is not a state function for generic active fluids, Nat. Phys. 11, 673 (2015).

[30] J. Smrek and K. Kremer, Small Activity Differences Drive Phase Separation in Active-Passive Polymer Mixtures, Phys. Rev. Lett. 118, 098002 (2017).

[31] J. Smrek and K. Kremer, Interfacial properties of active-passive polymer mixtures, Entropy 20, 520 (2018).

[32] J. Stenhammar, R. Wittkowski, D. Marenduzzo, and M. E. Cates, Activity-Induced Phase Separation and Self-Assembly in Mixtures of Active and Passive Particles, Phys. Rev. Lett. 114, 018301 (2015). 
[33] S. N. Weber, C. A. Weber, and E. Frey, Binary Mixtures of Particles with Different Diffusivities Demix, Phys. Rev. Lett. 116, 058301 (2016).

[34] A. Y. Grosberg and J.-F. Joanny, Nonequilibrium statistical mechanics of mixtures of particles in contact with different thermostats, Phys. Rev. E 92, 032118 (2015).

[35] E. Ilker and J.-F. Joanny, Phase separation and nucleation in mixtures of particles with different temperatures, Phys. Rev. Res. 2, 023200 (2020).

[36] A. Y. Grosberg and J.-F. Joanny, Dissipation in a system driven by two different thermostats, Polym. Sci. Ser. C 60, 118 (2018).

[37] M. Rubinstein, Polymer Physics (Oxford University Press, New York, 2003).

[38] Q. Huang, J. Ahn, D. Parisi, T. Chang, O. Hassager, S. Panyukov, M. Rubinstein, and D. Vlassopoulos, Unexpected Stretching of Entangled Ring Macromolecules, Phys. Rev. Lett. 122, 208001 (2019).

[39] T. C. O'Connor, T. Ge, M. Rubinstein, and G. S. Grest, Topological Linking Drives Anomalous Thickening of Ring Polymers in Weak Extensional Flows, Phys. Rev. Lett. 124, 027801 (2020).

[40] K. Kremer and G. S. Grest, Dynamics of entangled linear polymer melts: A molecular-dynamics simulation, J. Chem. Phys. 92, 5057 (1990).

[41] S. Plimpton, Fast parallel algorithms for short-range molecular dynamics, J. Comput. Phys. 117, 1 (1995).

[42] C. F. Abrams and K. Kremer, Effects of excluded volume and bond length on the dynamics of dense bead-spring polymer melts, J. Chem. Phys. 116, 3162 (2002).

[43] S. M. Pachong, I. Chubak, K. Kremer, and J. Smrek, Melts of nonconcatenated rings in spherical confinement, J. Chem. Phys 153, 064903 (2020).
[44] C. Svaneborg and R. Everaers, Characteristic time and length scales in melts of Kremer-Grest bead-spring polymers with wormlike bending stiffness, Macromolecules 53, 1917 (2020).

[45] See Supplemental Material at https://link.aps.org/supplemental/ 10.1103/PhysRevResearch.2.043249 for details on the rings' mean-squared internal distance profiles and autocorrelation functions of the squared gyration radius.

[46] M. Kardar and Y.-C. Zhang, Scaling of Directed Polymers in Random Media, Phys. Rev. Lett. 58, 2087 (1987).

[47] J. Smrek and A. Y. Grosberg, A novel family of space-filling curves in their relation to chromosome conformation in eukaryotes, Physica A 392, 6375 (2013).

[48] A. Rosa and R. Everaers, Conformational statistics of randomly branching double-folded ring polymers, Eur. Phys. J. E 42, 7 (2019).

[49] J. Smrek, K. Kremer, and A. Rosa, Threading of unconcatenated ring polymers at high concentrations: Double-folded vs time-equilibrated structures, ACS Macro Lett. 8, 155 (2019).

[50] K. A. Brakke, The surface evolver, Exp. Math. 1, 141 (1992).

[51] A. Rosa, J. Smrek, M. S. Turner, and D. Michieletto, Threadinginduced dynamical transition in tadpole-shaped polymers, ACS Macro Lett. 9, 743 (2020).

[52] I. W. Hamley and I. W. Hamley, The Physics of Block Copolymers (Oxford University Press, Oxford, 1998), Vol. 19.

[53] M. W. Matsen and R. B. Thompson, Equilibrium behavior of symmetric ABA triblock copolymer melts, J. Chem. Phys. 111, 7139 (1999).

[54] L. Leibler, Theory of microphase separation in block copolymers, Macromolecules 13, 1602 (1980). 